\title{
Agweddau ar Gwlt Martin o Tours mewn Llenyddiaeth Gymraeg hyd $c .1525$
}

Nid Martin o Tours (c.315/c.336-397) yw'r sant cyntaf y meddylir amdano wrth ystyried cwltiau'r seintiau yng Nghymru'r Oesoedd Canol. ${ }^{1}$ Ef oedd y gŵr o Pannonia (Hwngari, heddiw) a ddaeth yn efengylydd Gâl, ac ef oedd yr asgetig diymhongar a ddaeth yn esgob Tours. Ef, hefyd, oedd y milwr Rhufeinig ifanc a rwygodd ei fantell â'i gleddyf ger dinas Amiens, er mwyn rhoi hanner ohoni i ddyn tlawd. Ganrifoedd yn ddiweddarach, byddai'r brenhinoedd Carolingaidd yn cario 'mantell Martin' mewn brwydr, a daeth Martin yn un o nawddseintiau Ffrainc. ${ }^{2}$ Ysgrifennwyd buchedd Ladin Martin yn ystod ei fywyd, a'r testun hwn, Vita Sancti Martini gan Sulpicius Severus (c.360-?c.430), oedd un o'r bucheddau cynharaf a mwyaf dylanwadol yn y Gorllewin. ${ }^{3}$

O waith Sulpicius, yn bennaf, y tarddodd 'Buchedd Martin', fersiwn Cymraeg y ceir y copi cynharaf ohono mewn llawysgrif enwog yng nghasgliad Llyfrgell Genedlaethol Cymru a elwir yn LlGC 3026C neu Mostyn 88. ${ }^{4}$ Yn ôl coloffon ar ddiwedd y testun, fe'i copïwyd gan Gutun Owain yn 1488 ac enw'r cyfieithydd oedd 'John Trevor', sef, yn ôl pob tebyg, Siôn Trefor o Bentrecynfrig, mab Edward ap Dafydd o Fryncunallt. ${ }^{5}$ Lleolir Pentrecynfrig ym mhlwyf Llanfarthin neu St Martins yn Swydd Amwythig, nid nepell o'r Waun, ac roedd eglwys y plwyf wedi ei chysegru i Sant Martin er y ddeuddegfed ganrif os nad cynt. ${ }^{6}$ Y tebyg yw, felly, fod Siôn Trefor yn ystyried Martin yn nawddsant iddo. Roedd Gutun Owain yntau'n byw ym mhlwyf Dudlust, gerllaw, ac yn berchen tir ym mhlwyf Llanfarthin, ac mae'n debyg mai yn Llanfarthin y claddwyd ef. ${ }^{7}$ At hynny, credir i Gutun Owain greu'r llawysgrif ar gyfer trydydd gŵr lleol, yr ysgolhaig Siôn Edward o Blasnewydd, Gwernosbynt (ger y Waun), yntau'n berthynas i Siôn Trefor. ${ }^{8}$ Gellid ystyried y llawysgrif a'i chynnwys amrywiol yn ffrwyth ffyniant diwylliannol hirsefydlog gogledd-ddwyrain Cymru, a gynhelid gan ganolfannau eglwysig megis abaty Glyn-y-groes yn ogystal â diddordebau ysgolheigaidd uchelwyr lleol; ac fe all adlewyrchu, hefyd, ddatblygiadau gwleidyddol a deallusol ehangach y cyfnod arbennig hwn, a arweiniai'n ddiweddarach at y Dadeni Dysg. ${ }^{9}$ 
Mae'n drawiadol mai i'r gogledd-ddwyrain, hefyd, y perthyn y ddwy lawysgrif o'r unfed ganrif ar bymtheg sy'n cynnwys copïau o'r fuchedd hon, sef llawysgrif BL Add 14967, Llyfr Edward ap Roger o Riwabon, a luniwyd tua chanol y ganrif, a llawysgrif Cwrtmawr 530, a ysgrifennwyd gan Roger Morris o Goed-y-talwrn, Llanfair Dyffryn Clwyd, tua 1582. ${ }^{10}$ At hynny, ceir y copi cynharaf o fersiwn gwahanol, cryno iawn, o fuchedd Martin yn y casgliad pwysig o fucheddau seintiau a ysgrifennodd yr un Roger Morris yn llawysgrif Llanstephan 34, rhwng 1580 a $1600 .{ }^{11} \mathrm{Nid}$ ymddengys fod y fersiwn hwn yn dalfyriad o destun Siôn Trefor, ac fe allai fod yn gyfieithiad o bregeth Ladin, er nad yw'r union gynsail yn hysbys. ${ }^{12}$ Gellid ystyried y ddwy fuchedd, felly, yn dystiolaeth annibynnol am gryfder cwlt Martin yn y gogledd-ddwyrain.

Yr hyn sydd fwyaf trawiadol am fersiwn Siôn Trefor o'r fuchedd yw pa mor agos ydyw at ei gynsail Ladin. ${ }^{13}$ Wrth gyfieithu, ymddengys iddo wneud ei orau i gadw naws y testun gwreiddiol, heb ei newid yn fwriadol ac eithrio at ddibenion crynhoi, neu i wneud y cynnwys yn fwy dealladwy a pherthnasol i'w gynulleidfa. ${ }^{14} \mathrm{Nid}$ yw'r testun hwn yn dweud rhyw lawer wrthym, felly, am unrhyw neilltuolrwydd mewn agweddau Cymreig tuag at Martin yn yr Oesoedd Canol. Ond nid yw cyfeiriadau llenyddol Cymreig at Sant Martin yn gyfyngedig i'r bucheddau, nac i'r gogledd-ddwyrain, nac i'r cyfnod diweddar hwn. Trafodir isod, felly, gyfeiriadau eraill ato yn y rhyddiaith a'r farddoniaeth, gan geisio ateb y cwestiwn a oedd yna 'Martin (neu 'Marthin') Cymreig' drwy ystyried y gwahanol agweddau ar y sant a enynnodd ddiddordeb y beirdd a'r ysgrifwyr rhyddiaith, a chan dynnu rhai casgliadau ynghylch dosbarthiad daearyddol a datblygiad cronolegol ei gwlt. ${ }^{15}$

\section{Martin o Tours a'r delweddau ohono}

Yn ôl Vita S. Martini Sulpicius Severus, roedd Martin ('Martinus') yn frodor o Sabaria yn Pannonia (Szombathely yn Hwngari, erbyn heddiw). Fe'i gorfodwyd i fynd yn filwr yn y fyddin Rufeinig ond enillodd ei ryddid yn amser Iwlian Cesar ('Iwlian y Gwrthgiliwr'), a threulio cyfnod gyda St Hilari o Poitiers. Sefydlodd fynachlogydd ym Milan ac yn Ligugé, ger 
Poitiers, ac, ar ôl cael ei ethol yn esgob Tours, c.371, sefydlodd ei fynachlog enwocaf, Marmoutier, yn agos at y ddinas honno. Daeth yn enwog am ei fywyd asgetig, am ei genhadu dewr ac egnïol, ac am ei alluoedd yn iacháu ac yn bwrw cythreuliaid, ac fe'i hystyrir yn un o sefydlwyr mynachaeth y Gorllewin. Ymwelodd Sulpicius Severus â Martin yn 393 neu 394, ac mae'n debyg iddo orffen ei Vita yn 396, flwyddyn cyn i Martin farw. ${ }^{16}$ Ysgrifennodd destunau eraill amdano ar ffurf llythyrau (Epistulae) a thestunau ymddiddan (Dialogi), a'r rheini'n cael eu trosglwyddo gyda'r Vita ac, yn aml, gyda deunyddiau eraill am Martin. ${ }^{17}$ Byddai'r gweithiau hyn yn cael eu haddasu a'u cyfieithu wrth lunio fersiynau newydd o fuchedd Martin, mewn gwahanol gyfnodau ac at wahanol ddibenion, a lluniwyd nifer o destunau newydd, gwreiddiol, amdano ef a'i wyrthiau. ${ }^{18}$

Cyflwynodd Sulpicius bortread cymhleth a chyfoethog o Martin fel milwr, meudwy, abad, efengylydd ac esgob; fel gŵr dewr, asgetig, sanctaidd a gostyngedig; ac fel patrwm o sut i fyw a marw yn Gristion. Diau fod y darlun hwn yn adlewyrchu statws a dyheadau Sulpicius ei hun, ac yntau'n uchelwr breintiedig a oedd, fel eraill o'i gydnabod, wedi cael ei ysbrydoli i geisio efelychu asgetigrwydd Martin. ${ }^{19}$ Wrth i'w gwlt ddatblygu, câi Martin ei weld mewn gwahanol ffyrdd gan wahanol garfanau o bobl. Yn ôl Allan Scott McKinley, yn y ddwy ganrif ar ôl ei farwolaeth fe'i hystyrid yn esgob asgetig a sanctaidd, yn sefydlydd mynachaidd, yn sant ac esgob Tours, yn esgob yn fwy cyffredinol ('universal bishop'), yn iachäwr, ac yn eiriolwr dros eneidiau. ${ }^{20}$ Ac yn ôl Raymond Van Dam, fe'i hanrhydeddid fwyaf ar y dechrau fel patrwm o sut i fyw bywyd Cristionogol da, ac fel mynach, ond rhoddid mwy o bwyslais ar ei rym a'i awdurdod fel esgob wrth i'w gwlt gael ei hybu gan Perpetuus, esgob Tours, yn y bumed ganrif, a chan Gregori o Tours yn y chweched. ${ }^{21}$ Nid anghofid ei rôl fel mynach ac abad, er hynny; ysgrifennwyd pregethau amdano gan ddau arweinydd mynachaidd pwysig, sef Odo o Cluny (c.879-942) a Bernard o Clairvaux (1090-1153), ac roedd ganddo enw mawr yn Iwerddon fel un o sefydlwyr mynachaeth. $^{22}$

Erbyn diwedd yr unfed ganrif ar ddeg, daethai cwlt Martin dan reolaeth mynaich Marmoutier a chanoniaid Saint-Martin (sef yr eglwys yn Tours lle claddwyd ef); byddai'r cyntaf yn magu perthynas nawdd â chowntiau Blois ac Anjou, a'r ail yn gysylltiedig yn agos â brenhinoedd Ffrainc. ${ }^{23}$ 
Roedd storïau am wyrthiau Martin a'i allu fel eiriolwr ac fel iachäwr yn denu heidiau o bererinion i ymweld â'i fedd ac â Marmoutier; ${ }^{24}$ a phriodolid iddo'r gallu i amddiffyn rhag amrywiol beryglon megis stormydd a lladron, hefyd. ${ }^{25}$ Byddai'r brenhinoedd Carolingaidd yn cario 'mantell Martin' mewn brwydr, a chredid bod grym y sant wedi helpu achub Tours rhag y Llychlynwyr yn 903, a rhag y Tywysog Du yn $1356 .{ }^{26} \mathrm{Fe}$ 'i hystyrid yn un o nawddseintiau'r brenhinoedd Merofingaidd a'u holynwyr, ac er iddo gael ei ddisodli rywfaint gan Sant Denis yn amser y brenhinoedd Carolingaidd a Chapetaidd, cynyddodd ei statws eto yn ystod y bedwaredd ganrif ar ddeg. ${ }^{27}$

Diau fod delwedd Martin fel amddiffynydd milwrol yn gysylltiedig â'r cyfnod a dreuliodd yn y fyddin Rufeinig, er bod Sulpicius, yn ei Vita, yn pwysleisio bod Martin yn filwr mewn enw yn unig ar ôl iddo gael ei fedyddio. ${ }^{28}$ Yn ôl y Vita, dywedodd Martin ei hun, wrth ddadlau â Iwlian Cesar am gael ei ryddhau o'r fyddin, fod arno eisiau brwydro bellach yn achos Duw, a'i fod yn filwr Crist ('Christi ego miles sum'), ac adleisir y disgrifiad hwn ohono yn y naratif. ${ }^{29}$ Yn yr un modd, mae un o lythyrau Sulpicius yn disgrifio Martin yn defnyddio 'arfau gweddi' ('orationis arma'), a defnyddir ieithwedd filwrol debyg mewn perthynas ag ef mewn rhai ysgrifau diweddarach. ${ }^{30}$ Atgyfnerthid yr agwedd hon arno gan ei eiconograffi, ac yntau'n aml yn cael ei ddarlunio yn filwr ar gefn ceffyl, yn rhoi hanner ei fantell i'r dyn tlawd. ${ }^{31}$ Ond mewn achosion eraill, fe'i darlunnid yn esgob, a delwedd 'yr Esgob Martin' ('Martinus Episcopus') yn parhau i fod yn gryf yn Tours ac y tu hwnt. ${ }^{32}$

\section{Cwlt cynnar Martin ym Mhrydain}

Yn ôl Venantius Fortunatus, cydoeswr i Gregori o Tours, roedd y Brythoniaid ymhlith yr amrywiol bobloedd a garai Martin. ${ }^{33}$ Ac mae tystiolaeth arall fod Martin yn hysbys ym Mhrydain yn gynnar iawn. Gwyddys bod ei gyfaill Victricius, esgob Rouen, wedi dod yma am gyfnod byr (395-6) i dorri dadl yn yr Eglwys Brydeinig, ac mae'n bosibl iddo hybu mynachaeth ac efengylu 'Martinaidd' yn ystod ei ymweliad. ${ }^{34}$ Yn y rhan o'r ynys a adwaenid yn ddiweddarach fel Cymru, y tebyg yw bod dylanwad cynnar 
Martin ar ei gryfaf yn yr ardaloedd hynny lle roedd dylanwad y Rhufeiniaid yn gryf, sef y de-ddwyrain a'r gororau. ${ }^{35}$ Yn yr Oesoedd Canol cynnar gallai ei gwlt fod wedi cael ei atgyfnerthu gan gysylltiadau pellach â Gâl a gweddill y cyfandir, ac ag Iwerddon a Lloegr Eingl-Sacsonaidd, lle roedd yn sant pur adnabyddus. ${ }^{36}$ Diau i'r cwlt Cymreig gael hwb gyda dyfodiad y Normaniaid, yn enwedig wrth iddynt ymsefydlu yn ne Cymru o ddiwedd yr unfed ganrif ar ddeg ymlaen. ${ }^{37} \mathrm{~A}$ byddai cysylltiadau diweddarach â Lloegr a'r cyfandir wedi bod yn bwysig, fel y trafodir isod. Gallai hanes hir cysylltiadau o'r fath esbonio dosbarthiad y cysegriadau i Martin a'r enwau lleoedd sy'n cynnwys ei enw, a leolir gan fwyaf yn y de-orllewin, y de-ddwyrain a'r gororau. ${ }^{38}$

\section{Martin mewn rhyddiaith}

Y cyfeiriadau Cymreig cynharaf at Martin o Tours, mae'n debyg, yw'r ddau yn yr Historia Brittonum, testun y credir iddo gael ei ysgrifennu yn wreiddiol yng Ngwynedd yn 829-30. Dywedir bod 'sanctus Martinus' wedi dod yn enwog am ei alluoedd a'i wyrthiau ('claruit in virtutibus et signis') yn amser yr Ymerawdwr Maximus, ac iddo siarad ag ef; ac mewn adran arall o'r un testun, nodir eto fod 'Martinus Turonensis episcopus' yn enwog am ei alluoedd mawr ('magnis virtutibus'). ${ }^{39}$ Gallai'r awdur fod wedi cael yr wybodaeth am y sant a'i wyrthiau o gronicl Prosper o Acwitania, un o'i gynseiliau arferol, ond ni chyfeirir yno at Martin yn siarad â Maximus, felly mae'n bosibl fod y manylyn hwn wedi tarddu o Vita S. Martini Sulpicius, neu o'i Dialogi. ${ }^{40}$ Crybwyllir Martin, hefyd, yn un fersiwn o'r Annales Cambriae, sy'n nodi enwogrwydd yr esgobion 'beatus ambrosius et martinus'. ${ }^{41}$ Credir bod y testun hwn, sef y Cronicl Breviate (Testun-B), wedi ei ysgrifennu yn abaty Glyn-nedd yn ail hanner y drydedd ganrif ar ddeg. ${ }^{42}$

Ni chrybwyllir Martin yn ei gyd-destun hanesyddol ei hun yn Historia Regum Britanniae Sieffre o Fynwy, a ysgrifennwyd yn nhridegau'r ddeuddegfed ganrif. Ond, ar ôl adrodd hanes creu delw efydd o'r brenin Caduallo (Cadwallon) ar geffyl a'i gosod ar borth gorllewinol Llundain, dywedir bod eglwys wedi ei hadeiladu oddi tani a'i chysegru i Martin ('in honore 
sancti Martini'). ${ }^{43}$ Y tebyg yw mai eglwys St Martin, Ludgate, a olygir, a gall stori Sieffre adlewyrchu traddodiad cyfoes fod yr eglwys, a'i chysegriad i Martin, yn hynafol ${ }^{44}$ Hepgorwyd y manylyn hwn am y cysegriad o un cyfieithiad cynnar o waith Sieffre, sef 'fersiwn Llanstephan 1' o Brut y Brenhinedd, sy'n dyddio o tua chanol y drydedd ganrif ar ddeg ac a ysgrifennwyd, o bosibl, yn abaty Glyn-y-groes. ${ }^{45}$ Ond fe'i ceir yn Brut Dingestow, fersiwn y credir iddo gael ei lunio yng Ngwynedd yn ail hanner y ganrif honno. Nodir yma fod yr eglwys wedi ei chysegru 'yn anryded Seint Marthin'. ${ }^{46}$ Yn yr un modd, cyfeirir at y cysegriad 'yn enw duw a marthyn' yn 'fersiwn Cleopatra', a gysylltir â Glyn-y-groes ac a ymddangosodd, mae'n debyg, yn ail chwarter y bedwaredd ganrif ar ddeg; ac mae darlleniad tebyg ('yn enw duw a Marthin') yn y fersiwn diweddarach yn Llyfr Du Basing, yr ysgrifennwyd y rhan berthnasol ohono gan Gutun Owain. ${ }^{47}$

Y cyfeiriad gwreiddiol cynharaf at Martin a ganfuwyd mewn rhyddiaith Gymraeg yw'r un yn 'Buchedd Dewi', a gyfieithwyd o bosibl yn ail hanner y drydedd ganrif ar ddeg, er bod y copi hynaf sy'n goroesi, yn Llyfr Ancr Llanddewi Brefi (Rhydychen, Coleg yr Iesu 119), yn dyddio o 1346. ${ }^{48}$ Mae'r rhan fwyaf o'r fuchedd yn tarddu o Vita S. David Rhygyfarch, ond ymhlith yr ychwanegiadau yn y fersiwn Cymraeg ceir y frawddeg ganlynol: 'Ac yn y mod y rodes Duw Matheu yn Iudea, a Lucas yn Alexandria, a Christ yg Kaerussalem, a Pheder yn Rufein, a Martin yn Ffreinc, a Sampson yn Llydaw, y rodes y Dauid sant vot yn Ynys Prydein.' ${ }^{49}$ Pwrpas y frawddeg, wrth gwrs, yw hybu statws Dewi fel nawddsant cenedlaethol, ond mae'n arwyddocaol fod Martin wedi ei gynnwys ymhlith y ffigurau pwysig eraill y cyffelybir Dewi iddynt, a'i fod yn cael ei ddarlunio yn efengylydd neu nawddsant Ffrainc.

Ategir y statws hwn, o bosibl, gan gyfeiriad at Martin mewn cyfieithiad arall, sef atodiad i 'Cronicl Turpin', un o'r storïau sy'n ymwneud â Siarlymaen a'i ddilynwyr. Yn y testun byr hwn, a geir yn Llyfr Coch Hergest (1382x1405), enwir Martin unwaith eto ymhlith seintiau pwerus eraill, a hynny mewn araith sy'n gofyn am waredigaeth rhag cosb ddwyfol: "“O Duw y Cristonogyon, Duw Iago, Duw Meir, Duw Pedyr, Duw Marthin, Duw Hollgyuoethawc . ..". ${ }^{50}$ Ceir golwg wahanol, ysgafnach, ar y ffydd a roddid yng ngalluoedd Martin yn y stori am 'edyn Seint Martin' a 
adroddir yn 'Chwedlau Odo' (cyfieithiad a ddyddir i ail hanner y bedwaredd ganrif ar ddeg), a'r aderyn yn galw ar 'Duw tec a Seint Martin' i'w gynorthwyo wrth iddo gael ei ddychryn gan syrthiad deilen. ${ }^{51}$

Yn olaf, mae'n ddiddorol cymharu'r cyfeiriadau at 'Martin' neu 'Martinus' yn 'Chwedleu Seith Doethon Rufein', testun a geir yn Llyfr Coch Hergest ac mewn dwy lawysgrif arall a gysylltir â Morgannwg. ${ }^{52} \mathrm{Nid}$ yw cyd-destun hanesyddol y chwedl yn cyd-fynd â dyddiadau Martin o Tours, am iddi gael ei lleoli yn amser 'Diawchleison', sef yr Ymerawdwr Diocletian, a fu farw c.312. ${ }^{53}$ Serch hynny, mae'n werth ystyried a wyddai'r awdur am storïau am Martin yn ymwneud â gwahanol ymerawdwyr, ac a allai hyn fod wedi peri iddo ddewis 'Martin(us)' yn enw teilwng ar gyfer un o'r doethion. ${ }^{54}$

\section{Martin y beirdd canoloesol}

Daeth yr enw Mart(h)in yn weddol gyffredin mewn barddoniaeth yn ystod y bymthegfed ganrif, ond tri chyfeiriad, yn unig, a ganfuwyd o'r cyfnod cyn $c .1400 .{ }^{55}$ Ceir y cyfeiriad cynharaf, o bosibl, mewn awdl grefyddol gan Einion ap Gwalchmai, ac yntau'n un o weinidogion pwysicaf Llywelyn ab Iorwerth rhwng 1216 a $1223 .{ }^{56}$ Awgryma Rhian M. Andrews ei fod wedi ei eni tua 1153; felly fe allai iddo fod yn weithgar fel bardd erbyn degawdau olaf y ddeuddegfed ganrif. ${ }^{57}$ Fodd bynnag, mae cynnwys y gerdd, a'r bardd yn erfyn am faddeuant am bechodau bywyd hir a llawn, yn awgrymu ei bod yn waith gŵr oedrannus; felly'r tebyg yw iddi gael ei chanu yn negawdau cyntaf y drydedd ganrif ar ddeg. ${ }^{58}$ Cyfeiria'r bardd at Bedr (ceidwad allweddi teyrnas nef yn ôl Mathew 16.19) fel un a allai ei rwystro rhag cyrraedd ei 'addas' ('haeddiant') a'i '[d]reftad', ac at Fihangel yn ei hebrwng; a chrybwylla dri sant brodorol, sef Cwyfan (nawddsant Llangwyfan, Môn), Elfodd a Chenau, cyn galw ar nifer o seintiau eraill i eiriol drosto:

Mair a Sain Silin, Marthin, Mathau, Marcus a Lucas, Ionas enau,

Ar Dduw fy enaid, cyfraid cyfrau,

Eiriolwch, sefwch yn eich swyddau! $!^{59}$ 
Mae amheuon wedi eu codi ynghylch uniaethu'r 'Marthin' hwn â Martin o Tours, gan dynnu sylw at Martin arall, sef Llydawiad neu Frython a fu'n gyd-weithiwr i Sant Padrig. ${ }^{60}$ Gallai cysylltiadau Gwynedd ag Iwerddon, yn enwedig yn ystod amser Gruffudd ap Cynan a'i feibion, fod wedi helpu hybu cwlt y Martin hwn; ond ar y llaw arall, gallai'r un math o gysylltiadau fod wedi hybu cwlt Martin o Tours, ac yntau'n ddigon adnabyddus yn Iwerddon. ${ }^{61}$ At hynny, fel mae Huw Pryce wedi dangos, roedd cysylltiadau cynyddol rhwng llywodraethwyr Gwynedd a'r 'byd Eingl-Ffrengig' yn y drydedd ganrif ar ddeg, a'r llywodraethwyr hynny'n barod i fabwysiadu syniadau ac arferion estron. ${ }^{62}$ Ac wrth gwrs, roedd Llywelyn ab Iorwerth yn briod, er tua 1205, â Siwan, merch y Brenin John. Disgynnai hi o gowntiau Anjou, ac, fel y nodwyd uchod, roeddynt hwythau wedi bod yn noddwyr hael i hen fynachlog Martin yn Marmoutier, ger Tours. ${ }^{63}$ Mae digon o reswm, felly, dros gredu bod Martin o Tours yn sant y byddai bardd a gweinidog fel Einion ap Gwalchmai yn gwybod amdano ac yn dewis ei grybwyll mewn cerdd. Ac yn sicr, mae'r ffaith iddo grybwyll 'Marthin' ar ôl Mair a Silin (St Giles), a chyn y pedwar Efengylydd, yn awgrymu bod y 'Marthin' hwn yn sant rhyngwladol pwysig ac enwog. ${ }^{64}$

Gellir bod yn bur hyderus, felly, mai Martin o Tours a grybwyllir yng ngherdd Einion ap Gwalchmai. A'r tebyg yw mai ef yw'r 'Marthin' a grybwyllir mewn cerdd a ganodd bardd arall o Fôn, Gruffudd Gryg, tua chanol y bedwaredd ganrif ar ddeg. ${ }^{65}$ Mae'r cyd-destun yn debyg, a'r bardd yn crybwyll Pedr fel un a allai roi 'rhydid hyfedr rhad etifedd' ('r[h]yddid galluog etifedd gras'), cyn mynd ymlaen i ddisgrifio'r nef y mae'n gobeithio ei chyrraedd, a dweud ei fod yn dymuno bod 'Yn nhraed Serubin, yn rhòl Farthin, yn rhwyl ferthedd' ('Yn ôl traed [y] Sierwbim, yn rhòl Marthin, mewn gwychder disglair'). ${ }^{66} \mathrm{Fel} \mathrm{yr}$ awgryma Barry J. Lewis, ymddengys fod 'rhòl Farthin' yn cyfeirio at lyfr y bywyd, sef rhestr o'r sawl a achubir ar Ddydd y Farn ${ }^{67}$ Yn Llyfr Datguddiad, cysylltir llyfr y bywyd â'r 'Oen', sef Crist, ac ni ddaethpwyd ar draws unrhyw draddodiad sy'n priodoli i Martin o Tours swyddogaeth fel ceidwad y llyfr hwn. Mae'n debyg, fodd bynnag, nad oedd syniadau'r beirdd am restr neu ròl debyg yn gyfyngedig i gyd-destun y Beibl. Gellid cymharu llinellau gan Lewys Glyn Cothi, un o feirdd mwyaf y bymthegfed ganrif: 
Dau enw, er nad ŷnt unoed, yn fy rhol o nef y rhoed:

Henw Iesu fu ac a fydd

yn y dafl a henw Dafydd. ${ }^{68}$

Os gallai Lewys Glyn Cothi gyfeirio at ei '[r]ol o nef' ei hunan, efallai fod 'rhòl Farthin', yng ngherdd Gruffudd Gryg, yn dynodi rhestr o'r sawl a achubwyd drwy eiriolaeth Martin o Tours, neu restr o eneidiau teilwng yr oedd enw Martin ar ei brig. ${ }^{69}$

Yr unig gyfeiriad barddol arall at unrhyw Fart(h)in, o'r cyfnod cyn c. 1400, yw'r un yn 'Englynion y Clywaid':

A glyweist-di a gant Marthin,

Brenhinawl, sant y gyfrin?

'Namyn Duw nyt oes dewin'.70

Er nad oes sicrwydd pwy yw'r 'Marthin' hwn, Martin o Tours yw'r ymgeisydd amlycaf, fel y dywed Marged Haycock. ${ }^{71}$ Sant rhyngwladol arall, yr apostol Andreas, sy'n wrthrych yr englyn blaenorol; ac nid yw 'sant y gyfrin' ('sanctaidd ei gyfrinach', yn aralleiriad Haycock) yn ddisgrifiad anaddas o Martin o Tours, a'i Vita yn aml yn ei ddisgrifio'n gweld neu'n profi pethau a oedd yn guddiedig rhag ei ddilynwyr. ${ }^{72} \mathrm{O}$ ran y ddihareb, gellid ei chysylltu â gwyrthiau Martin, â'i allu i weld trwy ystrywiau cythreuliaid, neu â'i weithgareddau efengylaidd yng Ngâl, yn dymchwel delwau paganaidd, temlau a choed cysegredig. ${ }^{73}$ Fodd bynnag, am ei bod yn debygol fod y diarhebion yn rhagddyddio'r englynion, mae'n ansicr a oes rhyw lawer o arwyddocâd i'r priodoliad i Martin, y tu hwnt i'r ffaith fod ei enw yn odli â 'dewin'. ${ }^{74}$

Mae'r cyfeiriad at Martin fel un 'brenhinawl' yn fwy problemus, ar yr olwg gyntaf. Yn Vita S. Martini, dywed Sulpicius fod rhieni Martin 'heb fod o statws isel' yn ôl safonau'r byd, a dywed buchedd Siôn Trefor eu bod 'yn anrrydeddus o vrddas bydol'. ${ }^{75}$ Ond peth gwahanol yw dweud ei fod yn frenhinol. Gall mai gor-ddweud barddol sydd wedi codi statws y sant; roedd Cymry'r Oesoedd Canol yn hoff o gysylltu eu seintiau eu hunain â llinachau brenhinol, ac efallai i’r bardd dybio bod cyndeidiau 
Martin yr un mor bendefigaidd â rhai Dewi Sant, er enghraifft. ${ }^{76}$ Ond mae'n ddiddorol nodi bod tuedd cyffelyb ar waith yn Ffrainc, a bod chwedl a gyfansoddwyd gan fynaich Marmoutier yn y ddeuddegfed ganrif, 'Saith Cysgadur Marmoutier', yn honni bod Martin yn wyr i frenin Hwngari, ac yn perthyn i frenin Sacsonaidd ac ymerawdwr Rhufeinig. ${ }^{77}$ Mae'n ddigon posibl fod yr hanes hwn, neu, o leiaf, y traddodiad fod Martin o waed brenhinol, wedi cyrraedd Cymru erbyn adeg cyfansoddi'r englyn. Un dull amlwg o drosglwyddo deunydd o'r fath yw straeon pererinion; gwyddys bod nifer o Gymry, gan gynnwys beirdd a noddwyr, wedi teithio i ganolfannau pererindota mawr megis Rhufain a Santiago de Compostela. ${ }^{78} \mathrm{Ac}$ mae rhywfaint o dystiolaeth fod y beirdd yn ymwybodol o bwysigrwydd Martin o Tours yn y cyd-destun hwn, fel y trafodir isod.

Cyn gadael llinach Martin, rhaid nodi na chynhwyswyd ei achau yn 'Bonedd y Saint', ac ni welwyd unrhyw dystiolaeth fod y Cymry wedi ceisio ei gysylltu â llinachau'r seintiau brodorol. ${ }^{79}$ Ond mae un cyfeiriad arall yr ymddengys ei fod yn ymwneud â llinach y sant, a hwnnw mewn cerdd ofyn a ganodd Gutun Owain i Siôn ap Rhisiart, abad Glyn-y-groes, rhwng tua 1460 a 1470: 'Vn vonedd Marthin vynach, / Siôn, o Rywabon yw'r iach' ${ }^{80}$ Yr ystyr, mae'n debyg, yw bod yr Abad Siôn o'r un fath o linach â Martin, neu'n dod o wreiddiau cyffelyb, ond mae'n anodd gwybod a oedd gan y bardd linach neu wreiddiau uchelwrol mewn golwg, heb sôn am rai brenhinol. ${ }^{81}$ Fodd bynnag, mae'r cyfeiriad at Martin fel mynach yn ddiddorol, ac yn awgrymu y gwyddai Gutun rywfaint am hanes Martin o Tours cyn iddo gopio'r fuchedd. ${ }^{82}$ Efallai iddo glywed amdano yn eglwys Llanfarthin, neu drwy ei gysylltiadau â Siôn Trefor neu abaty Glyn-y-groes.

Diau fod Gutun Owain wedi tybio bod cyfeirio at Martin fel 'mynach' yn addas iawn mewn cerdd i abad, ac nid oes syndod fod Guto'r Glyn, cydoeswr hŷn iddo, yn cymharu un o'i noddwyr eglwysig yntau â'r sant. ${ }^{83}$ Gallai'r cyfeiriad hwn, hefyd, ddangos ymwybyddiaeth o yrfa Martin, a'r llinell 'Marthin yw'r Cyffin i'r côr' yn awgrymu bod y gwrthrych, y Deon Rhisiart Cyffin, yn arwain neu'n ysbrydoli'r gymuned eglwysig ym Mangor fel y gwnâi Martin yn ei fynachlog neu ei esgobaeth ef. Mae cerdd arall, gynharach gan Guto'r Glyn yn cynnwys yr unig gyfeiriad barddol y daethpwyd ar ei draws at Martin mewn perthynas ag iacháu, a hon yn agwedd arno a gâi gryn sylw yn Vita $S$. Martini a chan awduron diweddarach megis 
Gregori o Tours. ${ }^{84}$ (Y tebyg yw bod prinder y cyfeiriadau barddol at Martin fel iachäwr yn adlewyrchu prinder cymharol genre y cerddi iacháu; nid yw'n golygu bod yr agwedd hon arno yn llai hysbys i'r Cymry nag i eraill.$^{85}$ ) Gofynna Guto i naw sant, ac i’r Iesu, am eu cymorth i iacháu glin ei noddwr, Hywel ab Ieuan Fychan o Foeliwrch: 'Mair a'th ir â myrr a thus, / Marthin a fo cymhorthus' ${ }^{86}$ Er ei bod yn ddiddorol fod yr enw 'Mair' yn cael ei gydleoli â'r enw 'Marthin' eto, fel yng ngherdd Einion ap Gwalchmai, mae'n debyg mai Mair Fadlen, yn hytrach na'r Forwyn, a olygir y tro hwn. ${ }^{87}$ Wrth drafod y gwahanol seintiau a enwir, noda Eurig Salisbury, 'gall fod yn arwyddocaol y gellir cysylltu pedwar ohonynt ag eglwysi ar y Gororau nid nepell o Lansilin a'r pump arall ag eglwysi mewn dalgylch gyfagos', gan gyfeirio at Lanfarthin, ger y Waun, yn achos Martin. ${ }^{88}$

Gwahanol iawn yw cyd-destun cyfeiriad at Martin mewn cywydd gan Ddafydd ab Edmwnd, bardd arall o'r gogledd-ddwyrain, ac yntau'n gofyn i'r sant beri i ferch ymateb yn ffafriol iddo: 'Mae merch heb gytgam â mi, / Marthin, gwna help ym wrthi, / Arch roi ym Farged ferch Rys / . . . ${ }^{89}$ Diau fod tafod y bardd yn ei foch yma; nid oes dim yn hanes Martin o Tours sy'n awgrymu y byddai wedi cydymdeimlo â chais o'r fath. ${ }^{90}$ Efallai, yn wir, i Ddafydd ddewis crybwyll Martin am ei fod yn gwybod yn iawn ei fod yn enwog am ei ddiweirdeb; neu, fel arall, iddo ei ddewis oherwydd enwogrwydd ei alluoedd gwyrthiol; hynny yw, mai ef oedd y sant i droi ato pan oedd mawr angen cymorth. ${ }^{91}$

Nid oes syndod fod cyfeiriadau at Martin ymhlith cerddi toreithiog Lewys Glyn Cothi. Tyngir llw ar fedd y sant mewn cerdd fawl i Syr Wiliam Herbert, fel dull o gryfhau'r gymhariaeth rhwng Herbert ac Edward IV ar y naill llaw, a dau arwr Ffrengig, sef Rolant a'i frenin yntau, Siarlymaen, ar y llall: 'Siarlmaen yw Edwart, myn bedd Martin, / Rolant yw Herbart i rai haelion'. ${ }^{92}$ Efallai nad cyd-ddigwyddiad yw'r ffaith i'r bardd ddewis sant Ffrengig at y diben hwn. Tyngodd yr un bardd lw i'r sant ei hun ('myn Marthin') wrth ganmol noddwr arall, a defnyddir ymadrodd cyfatebol, 'ym Marthin', i gryfhau disgrifiad o alar mewn marwnad gan gydoeswr iddo, Hywel Dafi. ${ }^{93}$

Canodd bardd arall eto o'r un cyfnod, Huw Cae Llwyd, gerdd sy'n gofyn am fendith 42 o seintiau, a'r rhan fwyaf yn gysylltiedig â Brycheiniog, cyn iddo gychwyn ar ei daith i Rufain (yn 1475, yn ôl pob tebyg). ${ }^{94}$ Er na 
wyddys am eglwys wedi ei chysegru i Martin ym Mrycheiniog, awgryma Eurig Salisbury y gallai cysegriad rhyw allor neu gapel iddo fod wedi ei anghofio; neu, fel arall, fod statws Martin fel nawddsant eglwys Cwm-iou, ychydig dros y ffin, yn ddigon i sicrhau ei le yn y gerdd. ${ }^{95}$ Mae'n anodd dweud a ellir cysylltu'r cyfeiriad hwn ag enw mawr Martin fel amddiffynnwr, neu â'r ffaith fod ei fedd a'i fynachlog yntau yn ganolfannau pwysig i bererinion. Ond ceir awgrym cryfach o'r pethau hyn, neu'r cyntaf, o leiaf, yn 'Cywydd y Llong' Rhys Nanmor ( $f$. 1480-1513). ${ }^{96}$ Yma fe ymddengys fod y llong ar daith i Santiago de Compostela, a bod y bardd, wrth ei galw'n 'alarch Martyn farchog', yn hyderu bod nawdd y sant yn ei hamddiffyn. ${ }^{97}$ Mae'n ddiddorol cymharu emyn Lladin y credir iddo darddu o Iwerddon, mor gynnar â'r seithfed ganrif, a'r ymbiliwr yn gofyn am gymorth Duw i'w alluogi i deithio i gysegr Martin, gan ofyn i Martin ei hun ei amddiffyn rhag llongddrylliad ('per naufragium'). ${ }^{98}$ Ac yn wir, ceir yn un o Dialogi Sulpicius, ac yn y Legenda Aurea, hanes dyn a alwodd ar 'Dduw Martin' ('Deus Martini') pan oedd ei long mewn perygl, a'r môr yn tawelu ar unwaith. ${ }^{99}$

Mae'n bosibl fod arwyddocâd arbennig i'r gair 'marchog' yn 'Cywydd y Llong'; hynny yw, bod Martin yn cael ei ddarlunio yn 'filwr Duw'. ${ }^{100}$ Ond dyma'r unig gyfeiriad ato fel milwr a ganfuwyd yn y testunau Cymraeg, ac eithrio'r cyfeiriadau yn y ddau fersiwn o'i fuchedd. ${ }^{101}$ At hynny, ni chanfuwyd ond un cyfeiriad yr ymddengys iddo ymwneud yn benodol â digwyddiad enwocaf gyrfa filwrol Martin, sef yr achlysur pan roddodd hanner ei fantell i ddyn tlawd ger dinas Amiens. Mae'n drawiadol fod y cyfeiriad hwn mewn cerdd i neb amgen na Siôn Trefor o Wigynt, ym mhlwyf Llanfarthin, wyyr i'r Siôn Trefor a gyfieithodd fuchedd y sant. Ar ôl cyfeirio at letygarwch y noddwr adeg dathlu Gŵyl Martin, mae'r bardd, Huw ap Dafydd ap Llywelyn ap Madog ( $f$. 1520-1540), yn ei ganmol fel hyn: 'Mur a thangadwyn, Marthin godiad, / 'Mryd ei ddull am roi dy ddillad'. ${ }^{102} \mathrm{Yn}$ y llinell gyntaf, ystyr 'Marthin godiad', fe ymddengys, yw bod y noddwr yn debyg i Martin o ran ei gynnydd, ei linach neu ei wreiddiau; neu fe all fod y bardd yn tynnu sylw at ei fagwraeth o fewn plwyf y sant. ${ }^{103}$ Ac yn yr ail linell, cymherir Siôn â Martin o ran ei haelioni. Roedd rhoi dillad yn fotîff cyffredin yn y farddoniaeth, ond yma cysylltir yr haelioni yn benodol â duwioldeb a gostyngeiddrwydd Martin, a amlygwyd yn y weithred hael 
ger Amiens y ceir ei hanes yn ei fuchedd Gymraeg. ${ }^{104}$ Efallai fod y bardd yn talu teyrnged, fel petai, i gampwaith llenyddol taid ei noddwr, yn ogystal â dangos ei barch tuag at nawddsant y ddau. Ac efallai mai o'r testun hwnnw y dysgodd y stori hon am y sant, wrth ei glywed yn cael ei ddarllen yng nghartref ei noddwr neu yn eglwys y plwyf. Ni ellir bod yn sicr ynglŷn â hyn, fodd bynnag, am fod y stori mor gyffredin mewn gwahanol destunau, ac mewn darluniadau gweledol. ${ }^{105}$

Mae cyfeiriad arall at haelioni mewn perthynas â Martin mewn cerdd gan Lewys Môn ( $f$. c. 1480-1527), er na chrybwyllir dillad yn yr achos hwn. Dywed y bardd, ar ôl disgrifio haelioni'r noddwr gyda'i fwyd a'i win, 'Ofer i neb fu rhoi'n wych: / wedi Marthin, dim wrthych' ${ }^{106}$ Hynny yw, ar ôl dyddiau Martin, ofer fu rhoi unrhyw beth ('dim') yn 'wych' gan neb i gymharu â'r noddwr, a gyferchir yn uniongyrchol ('wrthych'); neu, mewn geiriau eraill, nid oedd neb wedi bod mor hael ag ef, er dyddiau Martin. ${ }^{107}$

A dychwelyd at Siôn Trefor o Wigynt, ceir cyfeiriad arall at Martin mewn cerdd a ganwyd iddo gan Forys ap Hywel ap Tudur, ac yntau'n dweud am Siôn a'i wraig, 'Yma 'dd oedd am y ddauddyn, / mae ei wyrth a'i nawdd, Marthin wyn'. ${ }^{108}$ Prin y gellid gofyn am fynegiad eglurach o rôl Martin fel nawddsant. Ond y mynegiad grymusaf oll o hyn, o bosibl, yw'r un ar ddiwedd marwnad a ganodd Tudur Aled ( $f$. 1495-1520) am Forgan ap Siôn ap Hywel Holand, o Eglwys-bach, Sir Ddinbych:

Crist, doe, yn croesi deuwr

Ym mhorth nef, Marthin a'i ŵr;

Mair yn borth, ym mron y bedd,

Aeth â'r gŵr i'w thrugaredd! ${ }^{109}$

Hynny yw, disgrifia Grist yn bendithio ('croesi') Martin 'a'i ŵr', sef Morgan, ym mhorth y nef, a'r Forwyn Fair hithau'n helpu Morgan i gael mynediad yno. Ymddengys, felly, fod Martin yn cael ei bortreadu fel yr un a hebryngai enaid y dyn marw i'r nef. Priodolid y swyddogaeth hon i'r Archangel Mihangel fel arfer, ond mae'n ddiddorol cymharu'r cyfeiriad yn 'Canu Dewi' Gwynfardd Brycheiniog at Ddewi yn arwain y Cymry at Dduw ar Ddydd y Farn, a Phadrig yn arwain ei luoedd yntau. ${ }^{110}$ Yn yr un modd, yng ngherdd Tudur Aled ymddengys fod Martin yn nawddsant arbennig 
i Forgan, a hynny, fwy na thebyg, am fod yr eglwys leol yn Eglwys-bach wedi ei chysegru iddo. Cofnodwyd y cysegriad hwn yn yr ymatebion i 'Parochial Queries' Edward Llwyd, tua diwedd yr ail ganrif ar bymtheg, ac, yn ôl arweinlyfr yr eglwys, ceir tystiolaeth amdano mor gynnar â 1530, yn ewyllys Owen Holand (brawd Morgan). ${ }^{11}$

Mae cyfeiriadau eraill at Martin yn yr un gerdd. Gallai'r llinellau 'Mae gar bron magwyr breiniaw / Mair, a than draed Marthin, draw', ddisgrifio lleoliad corff neu fedd y noddwr dan ddarluniau neu gerfluniau o Fair a Martin yn yr eglwys. ${ }^{112}$ Ac yn y cwpled 'Ba ddŵr hallt, ba wedd yr hin, / Ba ryferthwy bo Farthin?', ymddengys fod y sant yn galaru, naill ai fel arwydd o'i ofal tyner dros y gwrthrych, neu am ei fod yn cynrychioli teimladau'r plwyfolion. ${ }^{113}$

Mae'r gerdd hon gan Dudur Aled, felly, yn adlewyrchu gwedd leol ar y sant rhyngwladol hwn, ond fe all adlewyrchu, hefyd, ei fri ehangach fel eiriolwr pwerus. Mae'r ffaith fod y bardd yn crybwyll Martin ynghyd â'r Forwyn Fair, fel y gwnaeth Einion ap Gwalchmai, yn arwyddocaol oherwydd iddi hi y priodolid y gallu mwyaf oll i eiriol dros eneidiau, o ganlyniad i'w statws unigryw ymhlith lluoedd y nef. ${ }^{114}$ Ac nid y beirdd Cymraeg oedd y rhai cyntaf i bortreadu Mair a Martin yng nghwmni ei gilydd. Yn Dialogi Sulpicius, disgrifir Mair a'r seintiau Agnes a Thecla yn ymweld â Martin yn ei gell, a cheir gan Gregori o Tours ddisgrifiad o Fihangel yn ei dderbyn ymhlith yr angylion a Mair yn ei groesawu i'r nef. ${ }^{115}$ Yn fwy cyffredinol, darlunnid marwolaeth Martin yn batrwm o ddiwedd da i fywyd Cristion ar y ddaear. Yn ôl trydydd llythyr Sulpicius, rhagwelodd Martin ei hun y byddai 'mynwes Abram' yn ei dderbyn, ac yn yr ail lythyr, disgrifir gweledigaeth a gafodd yr awdur o'r sant yn esgyn i'r nef, ac un o'i ddisgyblion yn ei ddilyn, a mynegir yn eglur obaith Sulpicius y gallai yntau wneud yr un peth gyda chymorth gweddiau Martin. ${ }^{116}$ Roedd Martin fel pe bai wedi torri llwybr i'r nef ar gyfer y sawl a ddeuai ar ei ôl, ac, ar ôl cyrraedd yno, roedd yn eiriolwr pwerus dros eraill. ${ }^{117}$ Yn ei Vita, adroddir sut y bu i'w weddïau achub dyn a fu farw heb ei fedyddio rhag cael ei anfon i 'obscuris locis', neu 'leoedd tywyll' yng nghyfieithiad Siôn Trefor, ac yn yr Oesoedd Canol, hefyd, byddai rhai'n priodoli iddo'r gallu i ddwyn eneidiau allan o uffern. ${ }^{118}$ Nid oes rhyfedd, felly, fod Einion ap Gwalchmai yn troi at Martin am eiriolaeth, neu fod Gruffudd Gryg yn crybwyll 'rhòl Farthin' mewn 
perthynas â'r lle a ddymunai yn y nef, neu fod Tudur Aled yn ei ddarlunio yn hebrwng enaid Morgan Holand yno.

\section{Ĝ̂े $\mathrm{l}$ Martin}

Dethlir prif wyl Martin ar 11 Tachwedd, dyddiad ei angladd, ac mae ganddo whyl arall, sef 4 Gorffennaf, sy'n dathlu ei gysegru'n esgob ac sy'n cofnodi, hefyd, ddyddiad symud ei gorff i fan gorffwys newydd yn amser yr Esgob Perpetuus. ${ }^{119}$ Cofnodir gŵl 'Sancti Martini episcopi', ym mis Tachwedd, yng nghalendr y Vitae Sanctorum Wallensium, sy'n dyddio o c. 1200 ac yn tarddu o briordy Benedictaidd Trefynwy. ${ }^{120}$ 'Gŵyl Martin' yw hi yn un o'r calendrau Cymraeg cynharaf, yn llawysgrif BL Add 14912 (c. 1375xc. 1425); ${ }^{121}$ a cheir y cofnod Cymraeg cynharaf o'r ŵyl ym mis Gorffennaf ('Gŵwyl Marthin') mewn calendr a luniwyd tua'r un pryd, a rhan ohono wedi ei chadw yn llawysgrif Philadelphia $8680 .{ }^{122} \mathrm{Ni}$ chofnodir yr ŵyl hon mor aml â'r brif wŷl mewn calendrau diweddarach, ond mae'n werth nodi y ceir y ddwy wyl mewn calendrau a gopïwyd gan Gutun Owain; er enghraifft, yn llawysgrif LlGC 3026C, sy'n cynnwys copi Gutun o 'Buchedd Martin', cofnodir 'gwyl marthin esgob' ar 4 Gorffennaf a 'gwyl marthin' ar 11 Tachwedd. ${ }^{123}$

Mae 'Englynion y Misoedd' Dafydd ab Edmwnd yn gerdd ddiddorol sy'n debyg i galendr mydryddol, gan neilltuo englyn ar gyfer pob mis. Cofnodir prif wyl Martin yn yr englyn ar gyfer mis Tachwedd: 'Saint eneidiae llu santi Marthi / owdyl hyd Mundi'. ${ }^{124}$ Os deellir 'saint' yn enw lluosog yn hytrach nag yn ansoddair, gan roi coma ar ei ôl, ymddengys fod y llinell gyntaf yn cynnwys cyfeiriadau at Wyl yr Hollseint (1 Tachwedd) a Gŵyl yr Holl Eneidiau (2 Tachwedd), yn ogystal â Gŵyl Martin. ${ }^{125} \mathrm{O}$ ran 'llu santi Marthi', mae ffurf Ladinaidd y ddau air olaf, ac iddynt y terfyniadau '-i' a ddisgwylid ar gyfer geiriau genidol, o blaid eu cysylltu â'r 'llu'; ac eto, ni ellir sicrwydd, oherwydd gallai'r bardd fod wedi efelychu 'Sancti Martini' rhyw galendr Lladin heb feddwl am ystyr y terfyniad. ${ }^{26}$ Ystyr yr ail linell, mae'n debyg, yw bod sôn am Martin (a'r lleill hefyd, o bosibl) ar draws y byd. ${ }^{127}$ Ond, o ran y llinell gyntaf, a uniaethir yr holl eneidiau ffyddlon a'r holl seintiau â 'llu' Martin? Os felly, mae'n fynegiant 
cryf iawn o statws Martin yn y nef, ond nid yw'n anghydnaws â rhai o'r cyfeiriadau a drafodwyd uchod, yn enwedig 'rhòl Farthin' yng ngherdd Gruffudd Gryg, a darlun Tudur Aled o'r sant yn hebrwng noddwr marw i’r nef.

Roedd gwyliau'r seintiau yn rhan o fywyd beunyddiol yn yr Oesoedd Canol. Fe'u defnyddid i ddynodi dyddiad neu i osod terfynau amser, a cheir nifer o gyfeiriadau at wyl Martin yn y cyd-destunau hyn. Mae'r stori yn 'Chwedlau Odo' am ‘edyn Seint Martin' yn digwydd 'amgylch Gwyl Martin', er enghraifft, ac mae nifer o gyfeiriadau yn y croniclau, nad oes digon o le i'w rhestru yma. ${ }^{128}$ At hynny, roedd prif wyl y sant yn cael ei hystyried yn aml yn ddechrau'r gaeaf, fel a welir mewn traethawd ar hwsmonaeth sy'n rhoi'r cyngor, 'Dot dy deueit y my6n ty o wyl Martin hyt y Pasc'. ${ }^{129}$ Ac roedd arwyddocâd i'r ffaith ei bod yn dilyn cyfnod y cynhaeaf. Noda rhai fersiynau o'r Cyfreithiau y dylai gwŷr rhydd dalu treth (a fyddai wedi bod ar ffurf cynnyrch amaethyddol yn wreiddiol) i'r arglwydd rhwng Calan Tachwedd (neu Ŵyl yr Hollseint) a Gŵl Martin. ${ }^{130}$

Defnyddiai'r beirdd hwythau wyliau'r seintiau wrth gofnodi digwyddiadau pwysig. Ymddengys y gall y geiriau 'duedd gŵyl Marthin' mewn marwnad gan Lewys Môn gofnodi adeg angladd y gwrthrych, Nest Fechan, a 'duedd' o bosibl yn cynrychioli tywyllwch trosiadol, sef y tristwch a'r galar ar ei hôl, yn ogystal â nosweithiau tywyll mis Tachwedd. ${ }^{131}$ Defnyddir Gŵyl Martin at ddiben gwahanol mewn marwnad gan Ruffudd Hiraethog, sy'n ddiweddarach na'r testunau eraill a drafodir yma ond yn werth ei chrybwyll am fod ei gwrthrych, Syr Dafydd Owain o Faenan, yn dal bywoliaeth Eglwys-bach rhwng 1534 a $1558 .{ }^{132} \mathrm{Fel}$ y nodwyd uchod, roedd yr eglwys hon wedi ei chysegru i Martin, a chyfeiria'r bardd at ei ŵyl wrth gyferbynnu'r adeg hapus a llewyrchus cyn marwolaeth y gwrthrych â'r sefyllfa druenus ar ei hôl: 'Gwelais i gael sew a gwin, / Gwelaf wrthod gŵl Farthin'. ${ }^{133}$ Yr awgrym yw bod Syr Dafydd yn arfer rhoi gwledd i ddathlu gŵyl y sant, a cheir tystiolaeth am arfer tebyg yn un o'r cerddi i Siôn Trefor o Wigynt a grybwyllwyd uchod, wrth i Huw ap Dafydd ganmol ei letygarwch yntau: 'Ym mlaen y ford aml iawn fydd / Wyl Marthin ym win a medd'. ${ }^{134}$ 


\section{Ei enw}

Fel y gwelir o'r adrannau uchod, 'Marthin' yw'r ffurf fwyaf cyffredin ar enw'r sant yn y llenyddiaeth ganoloesol. Y tebyg yw i'r enw darddu o'r Lladin 'Martīnus'; disgwylid newid sain '-rt-' yn '-rth-' yn naturiol wrth fenthyg enw o'r Lladin. ${ }^{135}$ Yn wir, disgwylid newid y sain 'a' yn 'e', hefyd, drwy affeithiad- $i$, pe bai'r enw wedi ei fenthyg cyn tua diwedd y seithfed ganrif. ${ }^{136}$ Fodd bynnag, ni ellir sicrwydd o ran amseru'r benthyciad am y gallai'r sain 'a' fod wedi ei chadw drwy geidwadaeth, a'r enw efallai'n fwy cyfarwydd mewn ffynonellau Lladin ysgrifenedig nag yr oedd yn yr iaith lafar.

Y tebyg yw bod defnydd y ffurf 'Martin' yn un o'r calendrau Cymraeg cynharaf sydd wedi goroesi (yn llawysgrif BL Add. 14912), ac yn 'Chwedlau Odo', yn dangos effaith cyfieithu testun ysgrifenedig, a'r ysgrifydd heb newid dim ar y gair heblaw am hepgor y terfyniad Lladin. ${ }^{137}$ Ond dichon, hefyd, yr ystyrid y ffurf 'Martin' yn fwy safonol gan y sawl oedd yn gyfarwydd ag enw Lladin y sant, neu, yn enwedig yn ddiweddarach, y sawl a ddaethai ar draws yr enw 'Martin' yn Ffrangeg neu Saesneg. Gallai hyn esbonio defnydd y ffurf 'Martin' yn 'Buchedd Dewi' a chan Lewys Glyn Cothi, a'r ffurf 'Martyn' yng ngherdd Rhys Nanmor. Ond hyd yn oed yn y calendrau, ymddengys fod 'Marthin' yn fwy cyffredin na 'Martin', a'r ffurf gyntaf yn sicr a ffefrid gan y beirdd. Gall fod a wnelo hyn â'r ffaith fod 'Marthin' yn fwy cydnaws â seineg arferol y Gymraeg, ac felly'n fwy hydrin at ddibenion y gynghanedd. ${ }^{138}$ Ac i feirdd y gogledd-ddwyrain, wrth gwrs, byddai'r enw lle Llanfarthin wedi helpu sicrhau statws 'Marthin' fel enw safonol y sant.

Teitl y fersiwn cryno o fuchedd Martin yn Llanstephan $34 \mathrm{yw}$ 'Buchedd Marthin Escob', a chyfeirir at y sant, unwaith, fel 'Marthin Esgob' ym muchedd Siôn Trefor. ${ }^{139}$ Cyfeirir ato wrth enw tebyg mewn calendrau Cymraeg, weithiau, gan adlewyrchu eu cynseiliau Lladin, yn ôl pob tebyg. ${ }^{140}$ Ac yn yr un modd, gallai teitl y fuchedd fer fod wedi tarddu, yn y pen draw, o un o'r amrywiol fersiynau o deitl llawn Vita Sulpicius sy'n cyfeirio at Martin fel esgob. ${ }^{141}$ Fodd bynnag, ni ddaethpwyd o hyd i unrhyw achosion eraill o'r enw 'Mart(h)in Esgob' mewn llenyddiaeth ganoloesol Gymraeg, ac er cael 'Marthin vynach', 'Martyn farchog' a 'Marthin wyn' 
yn y farddoniaeth, nid oes ond enghreifftiau unigol o bob un. ${ }^{142} \mathrm{Nid}$ ymddengys fod gan Martin o Tours unrhyw epithet cyffredin iawn yn y Gymraeg, felly.

\section{Dosbarthiad daearyddol y cyfeiriadau}

Dengys y cyfeiriadau a drafodwyd uchod fod Llanfarthin yn ganolfan bwysig i gwlt Martin o Tours, yn y degawdau cyn ac ar ôl 1500, ac mae'n debyg fod Eglwys-bach yn ganolfan 'Martinaidd' arall yn y gogleddddwyrain, yn yr unfed ganrif ar bymtheg o leiaf. At hynny, gallai dylanwad lleol y sant fod wedi estyn cryn dipyn y tu allan i'r plwyfi hyn. Ymddengys fod Guto'r Glyn yn ei ystyried yn un o nawddseintiau lleol Hywel ab Ieuan Fychan o Foeliwrch, ger Llansilin, ryw naw milltir o eglwys Llanfarthin. Ac roedd Robert ap Rhys, y noddwr yr oedd ei haelioni'n debyg i eiddo Martin, yn ôl Lewys Môn, yn dod o Ysbyty Ifan, ryw bedair milltir ar ddeg i'r de o Eglwys-bach. Ond yn yr achos hwn, gallai cysylltiadau'r noddwr â Lloegr, fel caplan a chroesgludwr i'r Cardinal Wolsey, fod yn fwy arwyddocaol na lleoliad ei gartref yng Nghymru. ${ }^{143}$ Yn yr un modd, gallai cysylltiadau eglwysig fod wedi bod yn bwysicach na rhai lleol mewn perthynas â phenderfyniad Gutun Owain i gyfeirio at Martin wrth ganmol Siôn ap Rhisiart, abad Glyn-y-groes (llai na naw milltir o Lanfarthin). Ymddengys, fodd bynnag, fod Martin yn bur adnabyddus ymhlith beirdd mwyaf y gogledd-ddwyrain, ac yntau'n cael ei grybwyll nid yn unig gan Ddafydd ab Edmwnd a'i ddau ddisgybl, Gutun Owain a Thudur Aled, ond hefyd gan Guto'r Glyn, a gyfnewidiodd gywyddau dychan â Dafydd ac a farwnadwyd gan Gutun; at hynny, er na hanai Lewys Môn o'r ardal hon, roedd yn ddisgybl i Dudur Aled. ${ }^{144}$

Roedd Nest Fechan, gwrthrych marwnad Lewys Môn, yn byw ym Môn, felly gellid casglu o'r gerdd fod Gŵyl Martin yn cael ei dathlu yno (neu, o leiaf, yn ddyddiad adnabyddus yn y calendr) yn ei amser ef. Merch arall o Fôn oedd gwrthrych cerdd Dafydd ab Edmwnd, lle gofynnir am gymorth y sant i ennill ei ffafr, ac roedd Morgan Holand o Eglwys-bach, gwrthrych marwnad Tudur Aled, yn fab i ŵr a fu'n siryf Môn yn 1461. ${ }^{145}$ Gall fod yn berthnasol nodi, hefyd, mai o Loegr y daeth yr Holandiaid 
yn wreiddiol; ond, wrth gwrs, fe all mai cysegriad eglwys leol Morgan i Martin, yn unig, sy'n esbonio'r cysylltiad rhyngddo a'r sant a awgrymir yn y gerdd. Yn yr un modd, yn achos Rhisiart Cyffin, deon Bangor, fe all mai ei gysylltiadau eglwysig, yn hytrach na chysylltiadau lleol, a fu'n bennaf cyfrifol am benderfyniad Guto'r Glyn i'w gymharu â Martin. Mwy arwyddocaol, efallai, yw'r cyfeiriadau cynharach gan ddau fardd o Fôn, Einion ap Gwalchmai a Gruffudd Gryg; trafodwyd uchod y posibilrwydd fod a wnelo'r cyfeiriad yng ngherdd Einion â pholisïau a phriodas Llywelyn ab Iorwerth. Dylid nodi eto, hefyd, mai testun o Wynedd yw'r Historia Brittonum, a'i bod yn ymddangos bod gan y cyfieithydd a luniodd Brut Dingestow, testun arall a gysylltir â Gwynedd, fwy o ddiddordeb ym Martin nag yr oedd gan gyfieithydd fersiwn cynharach a grëwyd, mae'n debyg, yn abaty Glyn-y-groes. ${ }^{146}$ Serch hyn i gyd, mae'n anodd dweud i ba raddau yr ystyrid Martin yn nawddsant lleol yn y gogledd-orllewin, am na wyddys am eglwys a gysegrwyd iddo yno.

Yn ôl patrwm y cysegriadau a'r enwau lleoedd, disgwylid cryn dipyn o gyfeiriadau at Martin o'r de-orllewin a'r de-ddwyrain. Ac yn wir, gall un o'r cyfeiriadau cynharaf oll, yn 'Englynion y Clywaid', fod wedi tarddu o'r de neu'r canolbarth, a barnu wrth enwau'r seintiau eraill a grybwyllir. ${ }^{147}$ At hynny, gall y ffaith fod Huw Cae Llwyd yn crybwyll Martin ymhlith y seintiau a gysylltid â Brycheiniog ymwneud â chysegriad iddo rywle yn yr ardal; ac mae'n debygol fod Rhys Nanmor, y bardd a gyfeiriodd at long fel 'alarch Martyn farchog', yn byw yn Nhyddewi, er iddo hanu o Feirionnydd, a'i fod wedi canu'n bennaf i Syr Rhys ap Thomas o Abermarlais, 'prif gynorthwywr Cymreig y brenin Harri VII'. ${ }^{148}$ Nid annisgwyl, felly, yw gweld agwedd filwrol, sifalrïaidd ar Martin yn un o'i gerddi ef. Mae'n bosibl mai yn Nhyddewi, hefyd, neu Landdewi Brefi, y lluniwyd 'Buchedd Dewi', gyda'i chyfeiriad at Martin fel efengylydd neu nawddsant Ffrainc. ${ }^{149} \mathrm{Nid}$ oes cofnod am Martin mewn fersiwn o'r Annales Cambriae a ysgrifennwyd yn Nhyddewi, ond fe'i crybwyllir mewn un a luniwyd, mae'n debyg, yn abaty Glyn-nedd. A pherthyn i ardal Morgannwg hefyd, o bosibl, y mae 'Chwedleu Seith Doethon Rufein', gyda'i 'Martin(us)' a fu'n ymwneud â'r Ymerawdwr Diocletian. ${ }^{150}$ 


\section{Casgliadau}

Fel y nodwyd uchod, ni ellir bod yn gwbl sicr pwy a olygir ym mhob un o'r cyfeiriadau a drafodir yma, yn enwedig y rhai barddol cynharaf. Ac eto, ymddengys mai Martin o Tours yw'r gwrthrych tebycaf o lawer. Ni wyddys i ba raddau yr oedd y Martin arall a grybwyllwyd uchod, cydweithiwr i Sant Padrig, yn hysbys yng Nghymru. A hyd yn oed os oedd yn hysbys, a chwltiau'r ddau sant wedi cymysgu â'i gilydd, fel y gwnaethant yn Iwerddon, y tebyg yw y byddai nodweddion Martin o Tours wedi cymryd drosodd fwyfwy yn ystod yr Oesoedd Canol diweddarach, o ganlyniad i gysylltiadau cynyddol â Lloegr a'r cyfandir. ${ }^{151}$

Dylid ystyried, hefyd, y gallai'r Cymry fod wedi datblygu eu syniadau unigryw eu hunain am hanes a chymeriad Martin o Tours, dros amser, heb ddylanwad unrhyw sant arall o'r un enw. Ond mewn gwirionedd, y peth mwyaf trawiadol am y cyfeiriadau llenyddol a drafodwyd uchod yw pa mor debyg yw'r Mart(h)in Cymreig i Martin o Tours. Mor gynnar â dechrau'r drydedd ganrif ar ddeg, yn awdl Einion ap Gwalchmai, fe'i darlunnir yn eiriolwr pwerus, a hynny yng nghwmni'r Forwyn Fair a seintiau pwysig eraill. Ac mae 'rhòl Farthin' yng ngherdd Gruffudd Gryg, cyfeiriad posibl Dafydd ab Edmwnd at yr Holl Eneidiau a'r Hollseint fel 'llu santi Marthi', a darlun Tudur Aled o Martin fel hebryngwr noddwr marw at y nef, yn awgrymu bod iddo'r un math o statws arbennig ag a briodolid i Martin o Tours gan Sulpicius Severus ac arddelwyr ei gwlt diweddarach yn Tours. Yn wir, diau y byddai dilynwyr Martin yng Ngâl neu Ffrainc wedi adnabod yr holl agweddau ar y sant a fynegir yn y llenyddiaeth Gymraeg, ac yntau'n cael ei ddarlunio'n farchog, yn fynach, yn arweinydd eglwysig, yn efengylydd neu'n nawddsant i'w genedl, yn ddyn hael elusengar, ac yn un y gellid galw arno am iacháu, cymorth ac amddiffyn yn ogystal ag eiriolaeth dros eneidiau.

Un agwedd ar Martin na châi gymaint o sylw yng Nghymru ag y disgwylid, efallai, o'i chymharu â'i gwlt yn Lloegr a Ffrainc, oedd ei swyddogaeth fel esgob. ${ }^{152}$ Efallai nad oedd 'Martin Esgob', fel un a gynrychiolai awdurdod yr Eglwys, yn apelio at Gymry'r Oesoedd Canol, a hwythau, yn rhy aml, wedi gweld estronwyr yn cael eu dyrchafu i'r swyddi eglwysig uchaf. ${ }^{153}$ Mae'n drawiadol, hefyd, mai yn 'Buchedd Dewi', yn unig, y 
cysylltir Martin yn uniongyrchol â Ffrainc, er y gall cysylltiad o'r fath fod yn ddealledig yn achos y cyfeiriad mewn atodiad i 'Cronicl Turpin', ac yn y llinellau gan Lewys Glyn Cothi sy'n cydleoli ei enw ef ag enwau Rolant a Siarlymaen. At hynny, gall cyfeiriad Lewys Glyn Cothi at fedd Martin, a'r cyfeiriadau at y sant ei hun yng ngherddi Rhys Nanmor ac, efallai, Huw Cae Llwyd, ddangos ymwybyddiaeth o'i bwysigrwydd mewn perthynas â phererindota.

Nid tan ar ôl i Siôn Trefor gyfieithu'r fuchedd y ceir cyfeiriadau at Martin yn rhoi ei ddillad, neu at ei elusengarwch yn gyffredinol, ond mae'n amhosibl dweud i ba raddau yr ysbrydolwyd y rhain gan gynnwys y fuchedd Gymraeg. Gallent hwy, a nifer o gyfeiriadau barddol y degawdau rhagflaenol, fod wedi eu hysbrydoli, yn hytrach, gan yr un ffyniant yng nghwlt y sant yn y gogledd-ddwyrain ag a ysbrydolodd y fuchedd ei hun. Diau fod digonedd o wybodaeth am Martin yn hysbys ym mhlwyfi ei eglwysi, drwy gyfrwng pregethau ac, efallai, ddarluniadau gweledol. Ni wyddys am unrhyw ddarluniau neu gerfluniau canoloesol ohono yng Nghymru, nac yn Llanfarthin; ${ }^{154}$ ond, ac ystyried cymaint sydd wedi ei golli, mae'n bosibl iawn eu bod wedi bodoli unwaith. ${ }^{155} \mathrm{Yn}$ wir, gall cwpled anodd ei ddehongli o waith Tudur Aled awgrymu y bu darlun o Martin yn Eglwys-bach. ${ }^{156}$

Ymddengys, hefyd, fod dylanwad lleol y sant wedi estyn cryn dipyn y tu hwnt i ffiniau ei blwyfi, gan ymledu drwy rwydweithiau cymdeithasol megis rhai'r beirdd a'u noddwyr. Byddai Martin wedi bod yn ddigon adnabyddus, wrth gwrs, yn y canolfannau eglwysig yr ymwelai'r beirdd â hwy, megis Glyn-y-groes; ac yng Nghymru'n gyffredinol, gellid disgwyl y byddai ymwybyddiaeth ohono'n uwch ymhlith eglwyswyr ac ysgolheigion, ac ymhlith yr uchelwyr hynny a chanddynt gysylltiadau â Lloegr neu'r cyfandir. Mae'n bosibl fod cysylltiadau tebyg wedi cyfrannau at ddechrau neu adfywio cwlt Martin ym Môn yng nghyfnod Llywelyn ab Iorwerth neu ei ragflaenwyr; gellid cymharu awgrym Colin A. Gresham mai Llywelyn a fu'n gyfrifol am ailgysegru eglwys yng Nghricieth i Gatrin, santes ryngwladol a oedd yn boblogaidd iawn ar y pryd. ${ }^{157}$ Yng ngoleuni hyn, mae'n ddiddorol ystyried a allai Llywelyn, neu un o'i deulu neu'i ddisgynyddion, fod wedi bod yn gyfrifol am gysegriad Eglwys-bach i Martin. ${ }^{158}$

Hyd yn oed cyn adeg Llywelyn, roedd Martin yn hysbys i ryw raddau yng Nghymru. Gwyddai'r Lladinwyr, o leiaf, am ei briod le mewn hanes, 
fel y dengys yr Historia Brittonum a'r Annales Cambriae, a'r tebyg yw bod Vita S. Martini Sulpicius Severus, a gweithiau Lladin eraill a gyfeiriai at y sant, ar gael yn bur gynnar. ${ }^{159}$ Mae'n wir nad tan tua dechrau'r drydedd ganrif ar ddeg y ceir y cyfeiriad cynharaf ato fel sant y byddid yn troi ato am eiriolaeth, a hwnnw gan fardd o Fôn. Ond efallai nad oes syndod, mewn gwirionedd, ac ystyried mor brin yw'r dystiolaeth lenyddol, yn gyffredinol, cyn tua chanol y ddeuddegfed ganrif, a bod llawer mwy o farddoniaeth wedi ei chadw o Wynedd, yn y cyfnod hwn, nag o'r ardaloedd hynny yn y de a'r gororau lle gwyddys bod cysegriadau cynnar i Martin. Mae tystiolaeth y farddoniaeth ddiweddarach, fodd bynnag, yn awgrymu bod y sant yn adnabyddus mewn sawl ardal o'r gogledd a'r de, a'i gwlt yn arbennig o gryf, fel y disgwylid, ym mhlwyfi'r eglwysi a gysegrwyd iddo. ${ }^{160}$ Diau fod y cwlt wedi parhau i gael ei atgyfnerthu, drwy ei hanes, gan amrywiol gysylltiadau o'r tu allan i Gymru, gan gynnwys straeon pererinion a theithwyr eraill. Gallai'r rhain fod wedi trosglwyddo storïau a manylion newydd am Martin a darddodd o ganolfannau pwerus ei gwlt yn Tours a Marmoutier, gan gynnwys ei achau 'brenhinol', o bosibl. Ac efallai fod cysylltiadau o'r fath, hefyd, wedi tueddu i 'safoni' ei gwlt, i ryw raddau, fel na ddatblygodd nodweddion Cymreig unigryw, hyd y gellir dirnad.

Rhaid pwysleisio, wrth gwrs, nad Martin o Tours oedd y sant amlycaf neu fwyaf poblogaidd, yng Nghymru'r Oesoedd Canol. Hyd yn oed os oedd yn ddigon adnabyddus ymhlith beirdd a llenorion a'u noddwyr breintiedig, ac ymhlith plwyfolion eglwysi fel Llanfarthin ac Eglwys-bach, fe all fod llawer o Gymry eraill yn ei ystyried yn sant estron nad oedd o ddiddordeb iddynt; neu efallai fod rhai heb wybod mwy amdano na dyddiad ei brif ŵyl. Ond dengys y farddoniaeth fod Martin yn cael ei weld gan rai Cymry, o leiaf, nid yn sant rhyngwladol neu Ffrengig ond yn un lleol ac agosatynt; eu nawddsant hwy eu hunain a allai eu cynorthwyo ar y ddaear a hwyluso eu taith i'r nef. 


\section{Nodiadau}

1 Tarddodd yr erthygl hon o'm gwaith yng Nghanolfan Uwchefrydiau Cymreig a Cheltaidd Prifysgol Cymru yn golygu 'Buchedd Martin' ar gyfer prosiect Cwlt y Seintiau yng Nghymru, a ariennir gan Gyngor Ymchwil y Celfyddydau a'r Dyniaethau (AHRC, rhif grant AH/K00302X/1). Bydd y golygiad newydd ar gael ar wefan y prosiect, www. seintiaucymru. ac.uk, a chyfeirir ato isod fel 'BMartin' wrth ddyfynnu. Diolchaf i David Parsons, Martin Crampin, John Koch, Alaw Mai Edwards, Eurig Salisbury a Ken Dark am eu cyngor ar wahanol agweddau, a diolchaf yn arbennig i Ann Parry Owen a Jane Cartwright am eu cyngor ac am eu sylwadau ar yr erthygl hon. Rwyf yn ddiolchgar hefyd i Diana Luft am ei chyngor ar y calendrau ac am rannu ei nodiadau ymchwil ar y pwnc.

2 Un o'r enwau Lladin ar fantell yw capella, a maes o law daethpwyd i alw'r greirfa y cedwid mantell Martin ynddi yn capella hefyd. Dyna, yn y pen draw, yw tarddiad y gair Cymraeg capel, fel y nodir yn Bedwyr Lewis Jones, Yn ei Elfen (Llanrwst, 1992), t. 100.

3 Am gronoleg bywyd Martin a'i berthynas â Sulpicius, gw. Clare Stancliffe, St Martin and his Hagiographer (Oxford, 1983), yn enwedig tt. 1-6, 71-2, 119-33; The Oxford Dictionary of the Christian Church, goln F. L. Cross ac E. A. Livingstone, trydydd argraffiad, wedi ei ddiwygio (Oxford, 2005), tt. 1050, 15678; gw. hefyd ymdriniaethau Jacques Fontaine, Sulpice Sévère: Vie de Saint Martin, tair cyfrol (Paris, 1967-9); Régine Pernoud, Martin of Tours: Soldier, Bishop and Saint (San Francisco, 2006); Christopher Donaldson, Martin of Tours: the Shaping of Celtic Christianity, argraffiad newydd (Norwich, 1997). Ceir golygiadau o'r Vita yn Fontaine, Sulpice Sévère, a Carolus Halm, Corpus Scriptorum Ecclesiasticorum Latinorum, cyfrol I (Vienna, 1866), a chyfieithiad Saesneg gan y Parch. Alexander Roberts, 'The Works of Sulpitius Severus', yn P. Schaff a'r Parch. H. Wallace (goln), Nicene and Post-Nicene Fathers, ail gyfres, cyfrol XI, argraffiad newydd (New York, 2007), tt. 1-126.

4 Gellir gweld y llawysgrif ar 'Oriel Ddigidol' Llyfrgell Genedlaethol Cymru, www. llgc.org.uk/cy/darganfod/oriel-ddigidoll. Mae golygiad Evan J. Jones o'r fuchedd ('Buchedd Sant Martin', Bulletin of the Board of Celtic Studies, 4:3 (1928), 189207 a 4:4 (1929), 305-10; Buchedd Sant Martin (Caerdydd, 1945)) yn parhau i fod yn werthfawr, ac er dyfynnu o'r golygiad newydd ('BMartin') isod, cyfeirir at olygiad Jones (Buchedd) gan amlaf am nad yw rhaniadau adrannau 'BMartin' wedi eu penderfynu'n derfynol, adeg ysgrifennu'r erthygl hon.

5 Ar Siôn Trefor, gw. Morfydd E. Owen, 'Prolegomena i Astudiaeth Lawn o Lsgr. NLW 3026, Mostyn 88 a'i Harwyddocâd', yn I. Daniel, M. Haycock, D. Johnston a J. Rowland (goln), Cyfoeth y Testun (Caerdydd, 2003), tt. 349-84 (t. 351); nodyn Ann Parry Owen ar Edward ap Dafydd a'i deulu ar 'Guto'r Glyn.net', www. gutorglyn.net (cyrchwyd 30 Medi 2016), a 'BMartin', rhagymadrodd.

6 D. R. Thomas, Esgobaeth Llanelwy: A History of the Diocese of St. Asaph (London, 1870), t. 637; C. Neville Hurdsman, A History of the Parishes of St Martin's and 
Weston Rhyn (Wrexham, 2003), tt. 17-18. Roedd plwyf Llanfarthin yn rhan o esgobaeth Llanelwy hyd at ddechrau'r ugeinfed ganrif.

7 J. E. Caerwyn Williams, 'Gutun Owain', yn A. O. H. Jarman a G. R. Hughes (goln), A Guide to Welsh Literature 1282-c.1550, wedi ei ddiwygio gan D. Johnston (Cardiff, 1997), tt. 240-55 (t. 240). Nodir man claddu'r bardd yn llawysgrif LlGC 872D (Wrecsam 1; 1590-2), 'Pa le y claddwyd y prydyddion hyn ... Guttyn owain yn llan farthin' (dyfynnir o Report on Manuscripts in the Welsh Language, gol. J. Gwenogvryn Evans, dwy gyfrol (London, 1898-1910), cyf. II, tt. 359-60).

8 Daniel Huws, Repertory of Welsh Manuscripts (i'w gyhoeddi), d.e. Edward(s), John, I; Owen, 'Prolegomena', tt. 351-2; a gw. hefyd nodyn Ann Parry Owen ar Siôn Edward o Blasnewydd yn 'Guto'r Glyn.net'.

9 A. Parry Owen, 'Gramadeg Gwysanau (Archifdy Sir y Fflint, D/GW 2082)', Llên Cymru, 33 (2010), 1-31 (16); Owen, 'Prolegomena', t. 349; Glanmor Williams, Wales and the Reformation (Cardiff, 1997), t. 16.

10 Ar B[ritish] L[ibrary] Add[itional] 14967, un o'r llawysgrifau ychwanegol yng nghasgliad y Llyfrgell Brydeinig, Llundain, a Cwrtmawr 530, llawysgrif yng nghasgliad Cwrtmawr, Llyfrgell Genedlaethol Cymru, gw. Huws, Repertory.

11 Ar lawysgrif Llanstephan 34, yng nghasgliad Llanstephan, Llyfrgell Genedlaethol Cymru, gw. Huws, Repertory. Cyhoeddir trawsysgrifiad o'r fersiwn hwn o'r fuchedd ar wefan prosiect Cwlt y Seintiau.

12 'BMartin', rhagymadrodd; Paulus Grosjean, 'Gloria Postuma S. Martini Turonensis apud Scottos et Britannos', Analecta Bollandiana, 55 (1937), 300-48 (346).

13 Roedd y gynsail honno yn cynnwys Vita S. Martini Sulpicius Severus ynghyd â darnau eraill ganddo ef a chan Gregori o Tours (Buchedd, t. v; 'BMartin', rhagymadrodd). Trosglwyddid y deunyddiau hyn gyda'i gilydd yn aml; gw. E.-Ch. Babut, Saint Martin de Tours (Paris, 1912), t. 300.

'BMartin', rhagymadrodd.

Trafodir y ffurf Gymraeg 'Marthin' uchod tt. 19-20.

Am y ffynonellau y seiliwyd y crynodeb hwn arnynt, gw. n. 3 uchod.

17 Cf. uchod, n. 13. Isod, dyfynnir y Vita a'r Epistulae o olygiad Fontaine (tt. 248-345), a'r Dialogi o olygiad Halm (tt. 152-216).

18 Gw. 'BMartin', rhagymadrodd, a'r cyfeiriadau yno.

19 Stancliffe, St Martin, tt. 72-3.

20 A. S. McKinley, 'The First Two Centuries of Saint Martin of Tours', Early Medieval Europe, 14 (2006), 173-200. Cf. Sharon Farmer, Communities of Saint Martin: Legend and Ritual in Medieval Tours (Ithaca, 1991), t. 2 et passim, yn tynnu sylw at natur 'amlystyrol' ('polysemic') cwltiau a defodau'r seintiau.

21 R. Van Dam, Saints and their Miracles in Late Antique Gaul (Princeton, 1993), tt. 13-28. Yn ôl Sherry L. Reames ('Saint Martin of Tours in the "Legenda Aurea" and Before', Viator, 12 (1981), 131-64 (140)), portreadir Martin fel 'a powerful bishop, a wonder-worker sent by God' yng ngweithiau llenyddol Gregori o Tours. 
22 Reames, 'Saint Martin', 141-6; Kathleen Hughes, The Church in Early Irish Society (London, 1966), t. 76; Richard Sharpe, 'Palaeographical Considerations in the Study of the Patrician Documents in the Book of Armagh (Dublin, Trinity College MS 52)', Scriptorium, 36 (1982), 3-28 (5-6).

23 Farmer, Communities of Saint Martin, tt. ix, 5-6, et passim.

24 Ibid., tt. 5-6, et passim; Pernoud, Martin of Tours, t. 172.

25 Gw. Dialogi II (III), §§7, 14; a disgrifiadau Gregori o Tours o wyrthiau Martin yn ei Libri de virtutibus sancti Martini episcopi, I.2, I.34, I.36 (testun Lladin yn Gregorii Episcopi Turonensis Miracula et Opera Minora, goln B. Krusch a W. Levison, Monumenta Germaniae Historica, Scriptores Rerum Merovingicarum, cyfrol I, rhan ii, argraffiad newydd (Hannover, 1969); cyfieithiad Saesneg yn Van Dam, Saints, tt. 199-303).

26 Farmer, Communities of Saint Martin, tt. 30-4, 299.

27 Ibid., tt. 24-5, 82-3, 299; gw. hefyd Van Dam, Saints, tt. 20-8.

28 Vita, 3.6, 'solo licet nomine'; cf. 'BMartin', §7, 'yn dwyn henw marchoc' (Buchedd, t. $4,11.8)$.

29 Vita, 4.3, 4.9; cf. 'BMartin', §8, "'Mi a ryvelais gyd a thi; goddef y'm bellach ryvelu gyd a Duw ...”. . . Ac ni vynnodd Duw roddi amgen varvolaeth y dros i varchoc ef, ond gorvod ar y gelynion heb golli dim gwaed' (Buchedd, tt. 4-5).

30 Epistulae I, 15; a gw. y cyfeiriadau at arfau trosiadol Martin, ac ato ef ei hun fel rhyfelwr, mewn cerddi o Marmoutier a gyfansoddwyd, o bosibl, yn fuan ar ôl ei farwolaeth (Van Dam, Saints, tt. 309-11). Cf. geiriau cyntaf y fersiwn cryno o fuchedd Martin yn Legenda Aurea Jacobus de Voragine, casgliad poblogaidd o fucheddau a luniwyd yn y drydedd ganrif ar ddeg: 'Martinus quasi Martem tenens, id est bellum, contra vitia et peccata' ('Martinus is like Martem tenens, one who makes war, namely, against vice and sin'); gw. Jacobi a Voragine Legenda Aurea Vulgo Historia Lombardica Dicta, gol. Th. Graesse, ail argraffiad (Leipzig, 1850), adran CLXVI; The Golden Legend: Readings on the Saints, cyf. W. G. Ryan, argraffiad newydd (Princeton, 2012), adran 166. Credir bod yr enw 'Martin(us)' yn tarddu o enw Mars, duw rhyfel y Rhufeiniaid (Pernoud, Martin of Tours, t. 19).

31 Ceir darlun tebyg o Martin mewn nifer o furluniau canoloesol yn Lloegr; credir bod y cynharaf, yn Wareham, Dorset, wedi ei greu yn y ddeuddegfed ganrif; gw. E. Clive Rouse, Medieval Wall Paintings, pedwerydd argraffiad (Princes Risborough, 1991), tt. 48, 50.

32 Gw. Farmer, Communities of Saint Martin, tt. 235-41; cf. Karin E. Olsen, 'Beggar's Saint but no Beggar: Martin of Tours in Ælfric's Lives of Saints', Neophilologus, 88 (2004), 461-75. Am gyfeiriadau Cymraeg at 'Mart(h)in Esgob', gw. yr adran ar enw'r sant, uchod tt. 19-20, ac am yr eiconograffi, gw. Fernando a Gioia Lanzi, Saints and their Symbols: Recognizing Saints in Art and Popular Images (Minnesota, 2003), t. 105; Rosa Giorgi, Saints and their Symbols (New York, 2012), tt. 348-51. 
33 Grosjean, 'Gloria Postuma', 300.

34 Anthony Birley, The People of Roman Britain (Letchworth, 1979), t. 156; Jeremy K. Knight, 'In Tempore Iustini Consulis: Contacts between the British and Gaulish Churches before Augustine', yn A. Detsicas (gol.), Collectanea Historica: Essays in Memory of Stuart Rigold (Maidstone, 1981), tt. 54-62 (t. 55).

35 Cf. K. R. Dark, Civitas to Kingdom: British Political Continuity 300-800 (Leicester, 1994), t. 64; Hughes, Church in Early Irish Society, t. 29.

36 Nora K. Chadwick, 'Early Culture and Learning in North Wales', yn N. K. Chadwick, K. Hughes, C. Brooke a K. Jackson (goln), Studies in the Early British Church (Cambridge, 1958), tt. 29-120 (tt. 110-11); Hughes, Church in Early Irish Society, t. 32; P. A. Wilson, 'The Cult of St. Martin in the British Isles', The Innes Review, 19 (1968), 129-43; Donaldson, Martin of Tours, tt. 133-42; Knight, 'In Tempore Iustini Consulis'; Dark, Civitas, tt. 55-8.

37 J. E. Lloyd, A History of Wales from the Earliest Times to the Edwardian Conquest, trydydd argraffiad (London, 1939), tt. 392-403.

38 Gw. Wilson, 'Cult of St. Martin', 136, a'r adran am Martin yn y 'Seintiadur' ar wefan prosiect Cwlt y Seintiau.

39 Chronica Minora Saec. iv. v. vi. vii., cyfrol III, gol. Th. Mommsen, Monumenta Germaniae Historica, Auctorum Antiquissimorum, cyfrol XIII (Berlin, 1894), tt. 166 (\$26), 168 (\$29); Nennius: British History and the Welsh Annals, gol. John Morris (London, 1980), \$\$26, 29; cf. The Historia Brittonum: 3: The 'Vatican' Recension, gol. David N. Dumville (Woodbridge, 1985), §14 ('uirtutibus et miraculis claruit'), §18.

40 Chronica Minora, cyfr. III, tt. 166 (nn. 4-5), 168 (n. 7); cf. Vita, §20; Dialogi I (II), §6, Dialogi II (III), §\$11-13. Am y cofnod yng nghronicl Prosper, gw. Chronica Maiora, cyfrol I, gol. Th. Mommsen, Monumenta Germaniae Historica, Auctorum Antiquissimorum, cyfrol IX (Berlin, 1892), t. 461 (\$1175), 'Martinus episcopus Turinorum Galliae civitatis multis [miraculorum signis] clarus habetur'; ac ar y testun hwn fel un o gynseiliau'r Historia Brittonum, gw. ymhellach David N. Dumville, 'Some Aspects of the Chronology of the Historia Brittonum', Bulletin of the Board of Celtic Studies, 25:4 (1974), 439-45 (444); J. F. Matthews, 'Macsen, Maximus, and Constantine', Cylchgrawn Hanes Cymru, 11:4 (1983), $431-48$.

${ }^{41}$ Annales Cambriae: the B text, gol. Henry W. Gough-Cooper (Bangor, 2015), t. 17 (argraffiad ar lein, croniclau.bangor.ac.ukleditions.php.cy). Roedd Ambrosius yn esgob Milan ac yn gydoeswr i Martin, a cheir yng ngwaith Gregori o Tours hanes amdano'n cymryd rhan yn angladd Martin, drwy weledigaeth wyrthiol (Libri de virtutibus, I.5; cf. Buchedd, tt. 32-3). Crybwyllir Ambrosius yng nghronicl Prosper hefyd; gw. Chronica Maiora, cyfrol I, t. 461 (\$1173).

42 Ni chynhwysir y cyfnod Rhufeinig Diweddar yn y fersiwn cynharaf o'r Annales Cambriae sy'n goroesi, sef y Cronicl Harleian (Testun-A), felly nid oes syndod na cheir cofnod am Martin yno; ond mae'n werth nodi nad oes cofnod amdano, 
ychwaith (ac eithrio cyfeiriadau at ei wyyl), yn y cronicl Cottonian (Testun-C) a ysgrifennwyd yn Nhyddewi yn ail hanner y drydedd ganrif ar ddeg, ac sy'n dechrau, fel Testun-B, adeg creu'r byd. Gw. disgrifiadau Ben Guy o'r gwahanol fersiynau yn croniclau.bangor.ac.uk/chronicles.php.cy (cyrchwyd 29 Awst 2016). Geoffrey of Monmouth: The History of the Kings of Britain, gol. Michael D. Reeve, cyf. Neil Wright (Oxford, 2007), 201.508-12; cf. The Historia Regum Britannie of Geoffrey of Monmouth: 1: Bern, Burgerbibliothek, MS. 568, gol. Neil Wright (Cambridge, 1984), §201; The Historia Regum Britannie of Geoffrey of Monmouth: 2: The First Variant Version: a Critical Edition, gol. Neil Wright (Cambridge, 1988), §201.

44 Alfred John Kempe, Historical Notes of the Collegiate Church or Royal Free Chapel and Sanctuary of St. Martin-le-Grand, London (London, 1825), tt. 4-6; Simon Bradley a Nikolaus Pevsner, London: the City Churches, argraffiad newydd (New Haven, 2002), t. 103. Adeiladwyd yr eglwys bresennol ar y safle yn 1677-86 (ibid.).

45 Llawysgrif Llanstephan 1, t. 201, 'Ac a dana6 ed adeylassant eglwys' (dyfynnir o 'Rhyddiaith Gymraeg o Lawysgrifau'r 13eg Ganrif Fersiwn 2.0', goln G. R. Isaac, S. Rodway, S. Nurmio, K. Kapphahn a P. Sims-Williams (Aberystwyth, 2013), cadair.aber.ac.ukldspace/handle/2160/11163). Mae'r rhan hon o'r stori ar goll yn y fersiwn cynnar, anghyflawn, yn Llyfrgell Genedlaethol Cymru, llawysgrif Peniarth 44; gw. Brynley F. Roberts, 'Astudiaeth Destunol o'r Tri Chyfieithiad Cymraeg Cynharaf o Historia Regum Britanniae Sieffre o Fynwy' (Traethawd Ph.D. Prifysgol Cymru, Aberystwyth, 1969). Ac mae ar goll, hefyd, o fersiwn Peniarth 21 o'r Brut. Ar y gwahanol fersiynau, gw. Brynley F. Roberts, 'Brut y Brenhinedd', yn J. T. Koch (gol.), Celtic Culture: A Historical Encyclopedia, cyfrol I (Santa Barbara, 2006), tt. 298-9; Patrick Sims-Williams, Rhai Addasiadau Cymraeg Canol o Sieffre o Fynwy (Aberystwyth, 2011).

46 Brut Dingestow, gol. Henry Lewis (Caerdydd, 1942), t. 204, llau. 8-9 (xii.13), 'Ac adanav y gunaethpvt eglvys, a'e chysegru yn anryded Seint Marthin'. Er ei bod yn anodd dweud pa mor arwyddocaol yw cynnwys neu hepgor y cyfeiriad hwn at Martin, mae'n werth nodi bod fersiwn Dingestow, fel y cyfryw, yn fwy tueddol i aralleirio na fersiwn Llanstephan 1; gw. Brynley F. Roberts, 'Ystoriaeu Brenhinedd Ynys Brydeyn: a Fourteenth-century Welsh Brut', CSANA Yearbook 8/9 (2011), 217-27 (221).

47 Brut y Brenhinedd: Cotton Cleopatra Version, gol. John Jay Parry (Cambridge, Massachusetts, 1937), t. 213, 11. 16; Llyfr Du Basing (llawysgrif LlGC 7006D), t. 195, col. 2, 11. 24 (gellir gweld y llawysgrif ar 'Oriel Ddigidol' Llyfrgell Genedlaethol Cymru). Ar y fersiwn hwn, gw. ymhellach Sims-Williams, Rhai Addasiadau, tt. 15-16; Roberts, 'Ystoriaeu', 222-3.

48 Ar ddyddiad y cyfieithu, gw. ibid., 218; gthg. The Welsh Life of St David, gol. D. Simon Evans (Cardiff, 1988), t. liv, lle awgrymir dyddiad yn hanner cyntaf y bedwaredd ganrif ar ddeg. 
Welsh Life, t. 11, llau. 10-13.

50 Ystorya de Carolo Magno o Lyfr Coch Hergest, gol. Stephen J. Williams, argraffiad newydd (Caerdydd, 1968), t. 175, llau. 21-2; cf. Historia Karoli Magni et Rotholandi ou Chronique du Pseudo-Turpin, gol. C. Meredith-Jones (Genève, 1972), t. 245, llau. xxi-xxiii, 'O Deus Christianorum, Deus Iacobi, Deus Mariæ, Deus Petri, Deus Martini, Deus omnium Christianorum'. Er bod rhai llawysgrifau cynharach yn cynnwys 'Cronicl Turpin', ni cheir yr atodiad hwn ynddynt; gw. Williams, Ystorya de Carolo Magno, t. xiv. Am y gwahanol fersiynau, gw. y wefan 'Rhyddiaith Gymraeg 1300-1425', goln D. Luft, P. W. Thomas a D. M. Smith (2013), www.rhyddiaithganoloesol.caerdydd.ac.uk; ac ar y Llyfr Coch, gw. Huws, Repertory.

51 Chwedlau Odo, gol. Ifor Williams, ail argraffiad (Caerdydd, 1957), adran III, ac ar y dyddiad, gw. ibid., tt. xli-xlii. Ar y stori hon gan Odo o Cheriton (c.1185-c. 1246) a fersiynau tebyg iddi, gw. John B. Smith a Lillis Ó Laoire, "The Silly Treun": an Odd Bird Identified', Folk Life, 47 (2009), 51-7 (51-2).

52 Chwedleu Seith Doethon Rufein, gol. Henry Lewis (Caerdydd, 1967), t. 21, a llau. 7, 921, 1000.

53 Ibid., 11. 1, ac ar Diocletian, gw. The Oxford Classical Dictionary, goln S. Hornblower, A. Spawforth ac E. Eidinow, pedwerydd argraffiad (Oxford, 2012), tt. 453-4.

54 Ystyrir y chwedl hon yn gyfansoddiad gwreiddiol, seiliedig ar storïau poblogaidd rhyngwladol, a noda ei golygydd, Henry Lewis, mai yn y fersiynau Cymraeg yn unig y ceir y 'Martin(us)' hwn (Chwedleu Seith Doethon Rufein, tt. 26, 93). Gw. uchod am amryw gyfeiriadau at Martin a Magnus Maximus, a cf. yr adroddiadau amdano'n ymwneud â Iwlian (Vita, §4; Buchedd, tt. 4-5) a Valentinian (Dialogi I (II), §5), ac am frwdfrydedd gwraig Maximus drosto (ibid., §6). Cyfeirir at y tri ymerawdwr hyn yn fersiwn y Legenda Aurea o fuchedd Martin, hefyd (Jacobi a Voragine, tt. 742, 743-4, 745; Golden Legend, tt. 678-9, 680, 682).

55 Gellir bod yn bur sicr nad oes cyfeiriadau eraill ym marddoniaeth y cyfnod hwn am fod mynegeiriau cynhwysfawr ar gael; gw. Ann Parry Owen, 'Mynegai i Enwau Priod ym Marddoniaeth Beirdd y Tywysogion', Llên Cymru, 20 (1997), 25-45; idem, 'Mynegai i Enwau Priod yng Ngwaith Beirdd y Bedwaredd Ganrif ar Ddeg', Llên Cymru, 31 (2008), 35-89); Graham Isaac, Yr Hengerdd: Mynegeiriau Cyflawn (CD-ROM, Aberystwyth, 2001).

56 Yr unig gyfeiriad a allai fod yn gynharach na hwn yw'r un yn 'Englynion y Clywaid' (gw. isod, ac am ddyddiad y gerdd honno, gw. n. 70). Ar Einion ap Gwalchmai, gw. David Stephenson, The Governance of Gwynedd (Cardiff, 1984), tt. 98, 210; Gwaith Meilyr Brydydd a'i Ddisgynyddion, goln J. E. Caerwyn Williams et al. (Caerdydd, 1994), tt. 427-31.

57 R. M. Andrews, 'Golwg ar Yrfa Gwalchmai', Llên Cymru, 27 (2004), 30-47 (36).

58 Gw. Gwaith Meilyr Brydydd, tt. 456-7; Catherine McKenna, The Medieval Welsh Religious Lyric: Poems of the Gogynfeirdd, 1137-1282 (Massachusetts, 1991), tt. 32, 
68-9, 83-4, 108-9; N. G. Costigan, Defining the Divinity: Medieval Perceptions in Welsh Court Poetry (Aberystwyth, 2002), tt. 93-5. Martin arall, cyd-weithiwr Padrig, mae'n werth nodi nad oes cyfeiriadau ym marddoniaeth y cyfnod hwn at Badrig ei hun fel sant y byddai'r Cymry'n troi ato am eiriolaeth. (Dau gyfeiriad at Badrig, yn unig, a nodir yn Parry Owen, 'Mynegai i Enwau Priod ym Marddoniaeth Beirdd y Tywysogion', a'r ddau yn 'Canu Dewi' Gwynfardd Brycheiniog. Mae un yn ei ddisgrifio yn gadael Mynyw (i wneud lle i Ddewi), a’r llall yn cyferbynnu ei rôl yn arwain ei luoedd at y nef, ar Ddydd y Farn, â rôl Dewi'n arwain y Cymry; gw. Gwaith Llywelyn Fardd I ac Eraill o Feirdd y Ddeuddegfed Ganrif, goln Kathleen Anne Bramley et al. (Caerdydd, 1994), 26.38 a 238, a golygiad newydd Ann Parry Owen o’r gerdd, ar wefan prosiect Cwlt y Seintiau.) Cf. Patrick Sims-Williams, Irish Influence on Medieval Welsh Literature (Oxford, 2011), t. 336, lle nodir nad oes cyfeiriadau at arwyr Gwyddelig mewn barddoniaeth lys Gymreig tan tua chanol y bedwaredd ganrif ar ddeg.

65 Ymddengys fod Gruffudd Gryg yn byw yng nghantref Aberffraw; gw. Gwaith Gruffudd Gryg, goln Barry J. Lewis ac Eurig Salisbury (Aberystwyth, 2010), tt. 3-4. Daliai disgynyddion Einion ap Gwalchmai dir yn yr un rhan o'r ynys; gw. A. D. Carr, 'The Extent of Anglesey 1352', Transactions of the Anglesey Antiquarian Society and Field Club (1971-2), 150-272 (161-3, 165, 171, 243); Registrum Vulgariter Nuncupatum 'The Record of Caernarvon', gol. Henry Ellis 
(London, 1838), tt. 44-7, 76; Jennifer Day, “Elidir gwir, gwarant iawn - ganon": Elidir ap Gwalchmai ac Elidir Sais', Llên Cymru, 28 (2005), 53-99 (54-5, 96 (Ffigur 1)). O ran uniaethu 'Marthin' y gerdd hon â Martin o Tours, cf. nodyn Lewis, Gwaith Gruffudd Gryg, t. 183.

Gwaith Gruffudd Gryg, 10.31, 34; dyfynnir aralleiriad Barry J. Lewis mewn cromfachau, uchod.

67 Ibid., t. 183, a gw. Philipiaid 4.3, 'Clement a'm cydweithwyr eraill, sydd â'u henwau yn llyfr y bywyd', a Datguddiad 20.15, 'Pwy bynnag ni chafwyd ei enw'n ysgrifenedig yn llyfr y bywyd, fe'i bwriwyd i'r llyn tân'; cf. Datguddiad 3.5, 13.8, 17.8, 20.12, 20.15, 21.27, 22.19 (dyfynnir o Y Beibl Cymraeg Newydd (Swindon, 1988)). Gw. hefyd GPC Ar Lein (Canolfan Uwchefrydiau Cymreig a Cheltaidd Prifysgol Cymru, 2014, www.geiriadur.ac.uk, cyrchwyd 23 Medi 2016), d.g. rhol (b), a'r ystyron yn cynnwys 'sgrôl', 'rhestr, cofrestr', a 'llyfr', ac ibid. (o dan 'Cyfuniadau'), rhol, \&c., y bywyd 'book of life'.

68 Gwaith Lewys Glyn Cothi, gol. Dafydd Johnston (Caerdydd, 1995), 139.37-40; cf. ibid., 212.43-4 'Rhol wen a ysgrifennaf / o rif saint ar fis o haf'.

69 Cf. y cyfeiriad ym muchedd Columba sy'n awgrymu bod enw Martin ar ben rhestr o esgobion neu seintiau, mewn gweddi dros y meirw; gw. A. O. Anderson a M. O. Anderson (goln), Adomnán's Life of Columba (Edinburgh, 1961), t. 490; R. Sharpe (cyf.), Adomnán of Iona: Life of St Columba (Harmondsworth, 1995), t. 366.

70 Blodeugerdd Barddas o Ganu Crefyddol Cynnar, gol. Marged Haycock (Abertawe, 1994), 31.17a-c. Dyddiwyd y gerdd yn betrus i ddiwedd y ddeuddegfed ganrif neu ddechrau'r drydedd ganrif ar ddeg gan Ifor Williams, ond nododd Marged Haycock y gall fod yn ddiweddarach, gan fod deunydd diarhebol yn tueddu i fod yn geidwadol; gw. Ifor Williams a T. H. Parry-Williams, 'Englynion y Clyweit', Bulletin of the Board of Celtic Studies, 3:1 (1926), 4-21 (6-7); Blodeugerdd, t. 316. Rhaid fod y gerdd yn gynharach na $c .1400$, sef diwedd cyfnod floruit Hywel Fychan, ysgrifydd y copi yn llawysgrif Llanstephan 27 (Llyfr Coch Talgarth); gw. Huws, Repertory, d.e. Llanstephan 27 a Hywel Fychan.

71 Blodeugerdd, t. 331.

72 Gw., e.e., Vita, §§11, 21-4; Buchedd, tt. 10-11, 20-4.

73 Pwysleisir yn y bucheddau y dylid priodoli gwyrthiau'r sant i allu Duw; gw., e.e., ymateb gostyngedig Martin pan ofynnwyd iddo ddod i iacháu merch, 'BMartin', \$24, 'Synnv a wnaeth Marthin gan y geiriav hynn a'i nekav, gan ddywedud nad oedd ef mor deilwng ac y dangosai Dduw arwydd morr rhinweddol a hwnnw yrddo ef' (Buchedd, t. 15; tebyg yw Vita, 16.5), a Llanstephan 34, t. 321, llau. $2-3$, 'a Duw a wnaeth lawer o wyrthiau erddo ef'. Am Martin a chythreuliaid, gw. Vita, §\$6, 17-18, 21-4; Buchedd, tt. 6, 15-16, 20-4; ac am ei waith cenhadol, gw. Vita, $\S \S 13-15$; Buchedd, tt. 11-14.

74 Williams a Parry-Williams, 'Englynion y Clyweit', 6; ond gw. hefyd Haycock, Blodeugerdd, tt. 315-16. Ceir yr un ddihareb, heb y cysylltiad â 'Marthin', yn y 
farddoniaeth ddiarhebol a'r rhestr o ddiarhebion yn Llyfr Coch Hergest; gw. Early Welsh Gnomic and Nature Poetry, gol. Nicolas Jacobs (London, 2012), V.5c ('Kalan Gaeaf'), VIII.4c ('Gorwynion'), IX.61 ('Gossymdeith Llefoet Wynebclawr'); Diarhebion Llyfr Coch Hergest, gol. Richard Glyn Roberts (Aberystwyth, 2013), 11. 900.

75 Vita, 2.1, 'parentibus secundum saeculi dignitatem non infimis'; 'BMartin', §1 (Buchedd, t. 1, llau. 4-5).

76 Am achau Dewi, gw. 'Rhygyfarch's Life of St David', goln R. Sharpe a J. R. Davies, §§2, 68, yn J. W. Evans a J. M. Wooding (goln), St David of Wales: Cult, Church and Nation (Woodbridge, 2007), tt. 107-55; Welsh Life, t. 1; P. C. Bartrum, Early Welsh Genealogical Tracts (Cardiff, 1966), tt. 54-5 ('Bonedd y Saint').

77 Farmer, Communities of Saint Martin, tt. 167, 310-11.

78 Gw. Glanmor Williams, 'Poets and Pilgrims in Fifteenth- and Sixteenth-century Wales', Trafodion Anrhydeddus Gymdeithas y Cymmrodorion (1991), 69-98; Katharine K. Olson, "'Ar ffordd Pedr a Phawl”: Welsh Pilgrimage and Travel to Rome, c. 1200-c.1530', Cylchgrawn Hanes Cymru, 24:2 (2008), 1-40. Cf. y cyfeiriadau mewn cerddi gan Walchmai ap Meilyr Brydydd a'i fab Einion sy'n awgrymu eu bod hwythau wedi dymuno mynd i'r Tir Sanctaidd (Gwaith Meilyr Brydydd, 14.10, 67-72, 81-6, a 27.39n; gw. hefyd Henry Lewis (gol.), Hen Gerddi Crefyddol (Caerdydd, 1931), t. 214; McKenna, Medieval Welsh Religious Lyric, t. 119; Costigan, Defining the Divinity, tt. 88, 94; Andrews, 'Golwg ar Yrfa Gwalchmai', 42).

79 Cf. y cofnod byr, 'Marthin, gwr o Sarmania', yn 'Achau'r Saint', traethawd diweddarach a seiliwyd ar 'Bonedd y Saint', a'r copi cynharaf gan Elis Gruffydd, c.1527; gw. Bartrum, Early Welsh Genealogical Tracts, tt. 68-71 (\$40). Yn Iwerddon, roedd traddodiad fod Sant Padrig yn fab i chwaer Martin, neu i berthynas agos arall iddo, a'i fod wedi mynd i Tours a derbyn tonsur mynach ganddo; gw. The Tripartite Life of Patrick with Other Documents Relating to that Saint, gol. Whitley Stokes, dwy gyfrol (London, 1887), cyf. I, t. 25, a chyf. II, tt. 432-3, 560-1; J. Gwynn (gol.), Liber Ardmachanus (Dublin, 1913), tt. cclviii-cclx.

80 L'Oeuvre Poétique de Gutun Owain, gol. E. Bachellery (Paris, 1950-1), VIII.13-14; Catrin T. Beynon Davies, 'Cerddi'r Tai Crefydd' (Traethawd M.A. Prifysgol Cymru, Bangor, 1973), cerdd 51 a tt. 376-7.

81 Gw. GPC Ar Lein, d.g. bonedd (a) a (b).

82 Davies, 'Cerddi'r Tai Crefydd', t. 377. Copïodd Gutun y fuchedd yn 1488 yn ôl y coloffon ar ddiwedd y testun.

83 'Guto'r Glyn.net', 58.6.

84 'Guto'r Glyn.net', 92.51-2. Yn rhagymadroddion y cerddi yn 'Guto'r Glyn.net', cynigir y dyddiad ?c.1435xc.1450 ar gyfer y gerdd hon a'r 1480au ar gyfer y gerdd i Risiart Cyffin. Am Martin fel iachäwr, gw. Vita, §§7, 8, 16, 18, 19; Dialogi I (II), $\$ 4$ (cf. Buchedd, tt. 7-8, 14-15, 17-19); Libri de virtutibus, passim. Noda Farmer, Communities of Saint Martin, t. 6, y cysylltid bedd y sant yn eglwys Saint-Martin, yn arbennig, â gwyrthiau iacháu. 
85 Ar y genre, gw. Bleddyn Owen Huws, "Llawer dyn ... / Â chywydd a iachawyd": Guto'r Glyn yr Iachawr', yn D. Foster Evans, B. J. Lewis ac A. Parry Owen (goln), Gwalch Cywyddau Gwŷr: Ysgrifau ar Guto'r Glyn a Chymru'r Bymthegfed Ganrif (Aberystwyth, 2013), tt. 283-303.

86 'Guto'r Glyn.net', 92.51-2; a gw. nodiadau Eurig Salisbury ar y gerdd, ibid.

87 Gw. y nodyn esboniadol ar linell 51 yn 'Guto'r Glyn.net', a cf. llau. 25-8, 'Mair Fadlen yw Elen whyl, / Mae i'th warchad, maith orchwyl. / Eli meddyges Iesu / A wnaeth feddyginiaeth gu'. Ar Fair Fadlen fel 'meddyges Iesu', gw. ymhellach Jane Cartwright, Mary Magdalene \& her Sister Martha (Washington, D.C., 2013), tt. $26-8$.

88 Gw. y nodiadau esboniadol ar gerdd 92 yn 'Guto'r Glyn.net'.

89 Dyfynnir o Gwaith Dafydd ab Edmwnd, gol. Thomas Roberts (Bangor, 1914), XVII.1-3, gan ddiweddaru'r orgraff, ychwanegu atalnodi a newid rhai llythrennau'n briflythrennau. Ar y gair 'cytgam' ('cellwair, chwarae'), gw. GPC Ar Lein, d.g.

90 Cf. ei gyngor digyfaddawd i fynach a chyn-filwr a oedd yn colli cwmni ei wraig, yn Dialogi I (II), §11, a’i sylwadau ar ddiweirdeb, ibid., §10.

91 Cf. stori 'Chwedlau Odo' am aderyn yn galw ar Martin i'w amddiffyn (gw. uchod tt. 8-9).

92 Gwaith Lewys Glyn Cothi, 112.105-6.

93 Ibid., 197.29; Gwaith Hywel Dafi, i, gol. A. Cynfael Lake (Aberystwyth, 2015), 6.31. Am achosion eraill o dyngu llw i seintiau, i wrthrychau a gysylltid â hwy, neu i Dduw, gw. GPC Ar Lein, d.g. $m y n^{l}$ ac $y m^{3}$. Roedd ymadroddion sangiadol o'r fath yn ddefnyddiol iawn i feirdd, wrth greu cynghanedd, ac roedd Lewys Glyn Cothi yn arbennig o hoff ohonynt. Ymhlith y llwon niferus eraill yn ei gerddi ceir rhai i seintiau rhyngwladol a brodorol megis Iago (Gwaith Lewys Glyn Cothi, 51.19, 111.17), Pedr (ibid., 35.51, 51.57), Melangell (ibid., 149.15), Non (ibid., 154.3) a Derfel (ibid., 148.41, 183.17), a thyngir llw i feddau tri sant arall heblaw Martin, sef Llechid, Ffraid a Llonio (ibid., 54.39, 176.16, 210.41).

94 Eurig Salisbury, 'Ymgyrch Farchnata o'r Flwyddyn 1475: Moliant Huw Cae Llwyd i Seintiau Brycheiniog', Brycheiniog, 47 (2016), 51-70; crybwyllir Martin yn llau. 31-2 (t. 65), 'Saint Lidnerth a'n cyfnertho, / Saint Silin neu Farthin fo'. O ran y cydleoliad â Silin, cf. cerdd Einion ap Gwalchmai (gw. uchod), ond fe all nad oes rhyw lawer o arwyddocâd i hyn, ar wahân i'r ffaith fod yr enwau'n odli. Salisbury, 'Ymgyrch Farchnata', 56, a gw. y nodyn esboniadol ar y llinell berthnasol yng ngolygiad newydd Eurig Salisbury o'r gerdd ar wefan prosiect Cwlt y Seintiau. Y cyfeiriad cynharaf at gysegriad eglwys Cwm-iou i Martin a ganfuwyd yw'r un yn John Ecton, Thesaurus Rerum Ecclesiasticarum, ail argraffiad gan Browne Willis (London, 1754), t. 464. Mihangel yw enw'r nawddsant yn ôl Browne Willis, Parochiale Anglicanum (London, 1733), t. 186, ond mae'n bosibl fod hwn yn wall, a gywirwyd yn y gyfrol ddiweddarach. Diolchaf i David Parsons am yr wybodaeth hon. 
'Barddoniaeth Llawdden a Rhys Nanmor', Mary Gwendoline Headley (Traethawd M.A. anghyhoeddedig, Prifysgol Cymru, Bangor, 1938), cerdd 51; Williams, 'Poets and Pilgrims', 88. 'Barddoniaeth Llawdden a Rhys Nanmor', 51.63, a gw. y nodyn ar y llinell, ibid. Michael Lapidge, 'A New Hiberno-Latin Hymn on St Martin', Celtica, 21 (1990), 240-51 (tt. 243-4, ac atodiad, llau. 21-4).

Dialogi II (III) §14; Jacobi a Voragine, t. 744 (Golden Legend, t. 681); ceir gan Paulinus o Périgueux a Gregori o Tours storïau eraill am Martin yn achub pobl rhag boddi; gw. Farmer, Communities of Saint Martin, t. 24; Libri de virtutibus, I.2, II.17.

100 Cf. uchod, nn. 29-30.

101 Yno, cyfeirir ato fel 'marchoc vrddol' yn ogystal â 'marchoc'; gw. 'BMartin', §§3, 4, 7, 8 (Buchedd, tt. 2, 4-5); Llanstephan 34, t. 321, 11. 11, t. 322, 11. 2. Efallai fod cysylltiadau sifalrïaidd, uchelwrol y term cyntaf, yn arbennig, wedi apelio at y llenorion Cymreig. Yn y mannau cyfatebol yn Vita S. Martini, ceir naill ai'r gair 'miles' neu ryw air sy'n perthyn iddo (Vita, 2.5 ('militaribus'), 2.7 ('miles'), 3.6 ('militauit'), 4.9 ('milite')). Golygai 'miles' naill ai 'milwr' yn gyffredinol neu 'troedfilwr' mewn Lladin clasurol, ond yn yr Oesoedd Canol roedd ei ystyron yn cynnwys 'marchog' (Saesneg 'knight') hefyd; gw. Dictionary of Medieval Latin from British Sources, goln R. E. Latham, D. R. Howlett a R. K. Ashdowne (Oxford, 1975-2013), d.g.

102 Gwaith Huw ap Dafydd ap Llywelyn ap Madog, gol. A. Cynfael Lake (Aberystwyth, 1995), 27.53-4.

103 Gw. GPC Ar Lein, d.g. codiad (a) 'dyrchafiad . . . cynnydd . . . magwraeth, twf'.

104 'BMartin', $\S 5$, 6 (Buchedd, tt. 3-4); cf. Vita, §3. Adroddir yr un hanes yn y fuchedd fer yn Llanstephan 34. Disgrifir achlysur nodedig arall pan roddodd Martin ei ddillad yn Dialogi I (II), §1, ond nid yw'r stori hon wedi ei chynnwys yn y bucheddau Cymraeg.

105 Fe'i ceir, er enghraifft, yn y Legenda Aurea (Jacobi a Voragine, tt. 741-2; Golden Legend, t. 678), ac mewn homiliau neu bregethau Hen Saesneg (e.e. The Vercelli Homilies and Related Texts, gol. D. G. Scragg (Oxford, 1992), XVIII.47-66). Ar ddarluniadau gweledol o Martin, gw. uchod, n. 31, a'r adran olaf, isod. Gwaith Lewys Môn, gol. Eurys I. Rowlands (Caerdydd, 1975), XLIX.57-8. Diolchaf i Ann Parry Owen am y dehongliad hwn. Am 'wrth' yn yr ystyr '(o'i gymharu) â', gw. GPC Ar Lein, d.g. wrth 1(e).

Dyfynnir o Ifor Williams, 'Sion Trefor o Wigynt', Bulletin of the Board of Celtic Studies, 5:1 (1929), 40-4 (43), gan ddiweddaru'r orgraff, ychwanegu atalnodi a newid un llythyren yn briflythyren. Mae'n debyg mai ystyr 'gwyrth' yma yw 'gallu rhyfeddol' neu 'rhinwedd', gw. GPC Ar Lein, d.g.

109 Gwaith Tudur Aled, gol. T. Gwynn Jones (Caerdydd, 1926), LXXI.99-102. Roedd cartref Morgan yn nhrefgordd Pennant neu Bennant Erythlyn ym mhlwyf Eglwysbach; gw. Roy Kenrick a Norman Tucker, Eglwysbach: its Origins and History 
(Eglwysbach, 1998), t. [30]; P. C. Bartrum, Welsh Genealogies AD 1400-1500 (Aberystwyth, 1983), 'Holland 2'; a cf. cyfeiriad Tudur Aled at Forgan fel 'aer y Pennant' yn ei farwnad, 11. 82 .

110 Gwaith Llywelyn Fardd, 26.35-40, a gw. golygiad newydd Ann Parry Owen o'r gerdd ar wefan prosiect Cwlt y Seintiau. Ar Fihangel, gw. Oxford Dictionary of the Christian Church, t. 1089; a cf. isod, n. 115, a cherdd Einion ap Gwalchmai, a drafodir uchod.

111 "Parochialia: Being a Summary of Answers to "Parochial Queries in Order to a Geographical Dictionary, etc., of Wales"', gol. Rupert H. Morris, yn Archaeologia Cambrensis supplements (1909-11), i, t. 32, 'Marthin ydiw i Saint yr unved a'r dheg o'r gayav' (o dan 'Eglwys Vâch'). Dyfynnir yr ewyllys yn Kenrick a Tucker, Eglwysbach, t. [30]; cyfeirir at roi cwpan cymun i eglwys 'Saynt Martyn in Denbigh, land, where I was borne called Eglowes Vaght'.

112 Gwaith Tudur Aled, LXXI.29-30, ac ar y dehongliad, gw. t. 611.

113 Ibid., llau. 63-4, a gw. GPC Ar Lein, d.g. rhyferthwy 'ffrydlif, llifeiriant mawr, dilyw'.

114 Oxford Dictionary of the Christian Church, tt. 1053-5; Jane Cartwright, Feminine Sanctity and Spirituality in Medieval Wales (Cardiff, 2008), tt. 53-66; McKenna, Medieval Welsh Religious Lyric, tt. 125-8. I'r beirdd, wrth gwrs, byddai'r cyflythreniad rhwng y ddau enw, 'Mair' a 'Marthin', wedi bod yn rheswm ychwanegol dros eu cydleoli; cf. y cwpled gan Guto'r Glyn a drafodwyd uchod, sy'n cydleoli Martin a Mair Fadlen ('Guto'r Glyn.net', 92.51-2).

115 Dialogi I (II), §13; Libri de virtutibus, I.5; cf. 'BMartin', §52, ‘[M]arthin, yr hwnn a ddyrchavodd Mihangel a'r engylion i vyny, ac a erbynniodd Mair a chor o weryddon gyd a hi, a pharadwys yn llawen yn i atal ymysc saint' (Buchedd, t. 33, llau. 12-14).

116 Epistulae III, 16; Epistulae II, 3-18.

117 Cf. llinellau o farddoniaeth o eglwys Saint-Martin yn Tours sy'n disgrifio Martin yn galw pobl at y nef, ac yntau ei hun wedi cyrraedd yno ac wedi 'sancteiddio'r daith honno drwy'r sêr' ('Sidereum ingressus sanctificavit iter'); gw. Luce Pietri, La Ville de Tours du IV au VI Siècle: Naissance d'Une Cité Chrétienne, Collection de l'Ecole Française de Rome, cyfrol 69 (Rome, 1983), tt. 804-5 (cerdd 5); Van Dam, Saints, t. 312.

118 Vita, 7.6; 'BMartin', \$14 (Buchedd, t. 8, 11. 13); Farmer, Communities of Saint Martin, tt. 71, 103-4, 226-7.

119 Farmer, Communities of Saint Martin, tt. 22-3, 197, 222-32, 269, 294; gw. hefyd Libri de virtutibus, I.6; Buchedd, tt. 33-5.

${ }_{120}$ Silas M. Harris, 'The Kalendar of the Vitae Sanctorum Wallensium (Vespasian A xiv)', Journal of the Historical Society of the Church in Wales, 3 (1950), 3-53; gw. hefyd Kathleen Hughes, 'British Museum MS. Cotton Vespasian A. XIV ("Vitae Sanctorum Wallensium"): its Purpose and Provenance', yn Chadwick et al. (goln), Studies in the Early British Church, tt. 183-200. 
121 BL Add 14912, ff. 13r, ‘G6yl martin'; dyfynnir o’r wefan 'Rhyddiaith Gymraeg 1300-1425'.

122 Philadelphia, Library Company of Philadelphia, 8680.O, ff. 3v, 'G6yl Marthin' ('Rhyddiaith Gymraeg 1300-1425'); Huws, Repertory; Ben Guy, 'A Welsh Manuscript in America: Library Company of Philadelphia, 8680.O', National Library of Wales Journal, 36 (2014), 1-26.

123 LlGC 3026C, tt. 19, 23. Ar y calendr, gw. Owen, 'Prolegomena', tt. 357, 362. Cf. calendrau a gopïodd Gutun yn llawysgrifau Peniarth 27i (t. 7, 'gwyl marthin', t. 11, 'gwyl marthin') a Coleg yr Iesu 141 ('G. Marthin', mis Gorffennaf (mae'r adran ar gyfer mis Tachwedd ar goll); Report, cyf. II, t. 36). Yr wyl ym mis Tachwedd, yn unig, a gofnodir mewn calendr yn Peniarth 40, llawysgrif a ysgrifennwyd gan Lewys Glyn Cothi (1469x1500, 'G. saint martin escop'; Report, cyf. I, t. 375); ac felly hefyd yn y calendrau yn Peniarth 191 (canol y bymthegfed ganrif, 'G. varthin escob'; Report, cyf. I, t. 1019) a Coleg yr Iesu 22 (ail hanner y bymthegfed ganrif, 'Gwyl marthin'; Report, cyf. II, t. 38). Ar y llawysgrifau, gw. ymhellach Huws, Repertory.

Gwaith Dafydd ab Edmwnd, LXIII.41-2.

Gthg. nodiadau Thomas Roberts, Gwaith Dafydd ab Edmwnd, t. 126, sy'n cyfeirio at Ŵyl yr Eneidiau a Gŵyl Sant Martin yn unig, mewn perthynas â'r llinellau hyn.

Rhoddir enwau'r seintiau yn y cyflwr genidol yn rheolaidd, mewn calendrau Lladin; gw. Raymond Clements a Timothy Graham, Introduction to Manuscript Studies (Ithaca, 2007), t. 196.

Cf. GPC Ar Lein, d.g. awdl (b) 'stori', er na nodir tystiolaeth am yr ystyr honno cyn y ddeunawfed ganrif. Chwedlau Odo, adran III.

Mae'r testun hwn, a gedwir yn Llyfr Coch Hergest, yn gyfieithiad o draethawd Eingl-Normaneg a ysgrifennwyd gan Walter o Henley yn y drydedd ganrif ar ddeg; gw. Alexander Falileyev, Welsh Walter of Henley (Dublin, 2006), tt. ix-xvi, 7 (XXXV.203-4), 140.

130 The Latin Texts of the Welsh Laws, gol. Hywel David Emanuel (Cardiff, 1967), t. 139, 'Quisquis liber ea que domino suo debet reddere inter kalendas Novembris et festum Sancti Martini reddat' (testun 'Latin A'); ibid., t. 390, '. . . inter festum Omnium Sanctorum et festum Sancti Martini' (testun 'Latin D'); Llyfr Blegywryd, goln S. J. Williams a J. Enoch Powell (Caerdydd, 1942), t. 83, llau. 23-5, 'Pob gwr ryd, rwg Gwyl Holseint a Gwyl Marthin y tal y'r arglwyd yr hyn a dylyho y talu'.

131 Gwaith Lewys Môn, XXIV.57.

132 'Y Bywgraffiadur Ar-lein', yba.llgc.org.uk/cy/index.html (cyrchwyd 30 Medi 2016), d.e. 'Syr' Dafydd (David) Owain; J. E. Griffith, Pedigrees of Anglesey and Caernarvonshire Families (Bangor, 1914), t. 196; Gwaith Gruffudd Hiraethog, gol. D. J. Bowen (Caerdydd, 1990), t. 521. 
133 Ibid., 84.71-2.

134 Gwaith Huw ap Dafydd, 27.51-2.

135 Henry Lewis, Yr Elfen Ladin yn yr Iaith Gymraeg (Caerdydd, 1943), t. 21; Kenneth Jackson, Language and History in Early Britain (Edinburgh, 1953), tt. $570-1$.

136 Gw. Jackson, Language and History, tt. 616-17; Lewis, Elfen Ladin, tt. 2, 27.

137 Gw. yr adran flaenorol, a Chwedlau Odo, adran III ('edyn Seint Martin'); cf. Les Fabulistes Latins, gol. Léopold Hervieux, cyfrol IV (Paris, 1896), tt. 183-4 (adran VII, 'De Quadam Ave Sancti Martini').

138 O'r ddau achos o 'Martin'/'Martyn' y daethpwyd ar eu traws mewn barddoniaeth, mae'r naill heb gyfrannu at y gynghanedd, a'r llall yn creu cynghanedd lusg gydag enw estron arall, 'Edwart' ('Barddoniaeth Llawdden a Rhys Nanmor', 51.63, 'Clyw, alarch Martyn farchog'; Gwaith Lewys Glyn Cothi, 112.105, 'Siarlmaen yw Edwart, myn bedd Martin'). Mae'n werth nodi bod Lewys Glyn Cothi yn defnyddio'r ffurf 'Marthin' mewn cerdd arall (ibid., 197.29, 'taro wrtho, myn Marthin').

139 'BMartin', $\$ 50$ (Buchedd, t. 32, 11. 4); mae'r geiriau 'Marthin Esgob' yn trosi 'Martinus episcopus', yn Libri de virtutibus, I.4.

140 Gw. n. 123, uchod, a hefyd C. R. Cheney (gol.), A Handbook of Dates for Students of British History, argraffiad newydd, wedi ei ddiwygio gan Michael Jones (Cambridge, 2000), tt. 78, 92.

141 Gw. Vita, \$1 (nodiadau Fontaine, t. 250), am amrywiol deitlau gan gynnwys 'Vita sancti Martini episcopi'; weithiau ychwanegid y geiriau 'et confessoris'. Nid oes teitl gan fuchedd Siôn Trefor, ond gallai ei geiriau cyntaf, 'Marthin Sant oedd esgob a chonffesor i Grist' ('BMartin', §1), fod wedi tarddu o deitl Lladin tebyg. Cf. y teitl 'De sancto Martino episcopo', yn y Legenda Aurea, a theitl testun Gregori o Tours, 'Libri de virtutibus sancti Martini episcopi'.

142 Gw. y cerddi gan Gutun Owain, Rhys Nanmor a Morys ap Hywel ap Tudur a drafodwyd uchod. Mae'r epithet 'verthyr' yn dilyn enw Martin yn y fersiwn o 'Englynion y Misoedd' Dafydd ab Edmwnd yn llawysgrif BL Add 14986, ff. 6v (1552), ac er bod hwn yn llurguniad o'r darlleniad gwreiddiol, yn ôl pob tebyg (gw. yr adran ar Wyl Martin, uchod), fe all adlewyrchu syniadau'r ysgrifydd am y sant. Nid oedd Martin yn ferthyr yn yr ystyr arferol, ond dywedodd Sulpicius y gellid ei ystyried yn ferthyr o ran dymuniad ac am iddo gosbi ei gnawd a dioddef dros ei ffydd (Epistulae II, 8-12); cf. brawddeg gyntaf testun y Legenda Aurea.

143 Rowlands, Gwaith Lewys Môn, t. 455.

144 'Y Bywgraffiadur Ar-lein', d.e. Dafydd ab Edmwnd, Gutun Owain, Lewis Môn, Tudur Aled; 'Guto'r Glyn.net', cerddi 66, 67, 68a, 68, 68b, 126; ac ar gysylltiadau Guto'r Glyn â'r ardal, gw. Eurig Salisbury a Barry J. Lewis, 'Guto'r Glyn: A Life', yn 'Guto'r Glyn.net'.

145 Bartrum, Welsh Genealogies AD 1400-1500, 'Holland 2'; 'Y Bywgraffiadur Arlein', d.e. Holland (teuluoedd). 
Dylid nodi, fodd bynnag, fod y cyfeiriad at Martin wedi ei gynnwys mewn fersiwn diweddarach a gysylltir â Glyn-y-Groes, ac mewn fersiwn a gopïwyd gan Gutun Owain. Haycock, Blodeugerdd, t. 316. Gw. Evans, Welsh Life, t. liv.

Fel y trafodwyd uchod, ni ellir ystyried hwn yn gyfeiriad dilys at Martin o Tours, ond fe all adlewyrchu ymwybyddiaeth yr awdur ohono.

151 Ar y cymysgu rhwng y ddau sant yn Iwerddon, gw. Baring-Gould a Fisher, Lives of the British Saints, cyf. III, tt. 439-40.

152 Gw. Farmer, Communities of Saint Martin, tt. 235-41, 274-5; Olsen, 'Beggar's Saint but no Beggar'; Reames, 'Saint Martin', 140, 153.

153 Glanmor Williams, Religion, Language and Nationality in Wales (Cardiff, 1979), tt. 39-41; idem, Wales and the Reformation, tt. 13-16.

154 Diolchaf i Martin Crampin am drafod yr eiconograffi â mi, ac i’r Parch. Stuart Jermy am yr wybodaeth am eglwys St Martin o Tours, Llanfarthin.

155 Noda Madeleine Gray fod seintiau rhyngwladol yn cael eu portreadu yn llawer mwy aml na seintiau brodorol, yn y diwylliant gweledol sy'n goroesi; gw. Images of Piety: the Iconography of Traditional Religion in Late Medieval Wales, British Archaeological Reports British Series 316 (Oxford, 2000), t. 25. Cf. nodyn T. Gwynn Jones, Gwaith Tudur Aled, t. 611.

157 C. A. Gresham, 'Tre Ferthyr and the Development of Cricieth', Caernarvonshire Historical Society Transactions, 27 (1966), 5-36 (6-8); gw. hefyd M. A. Ward, 'St. Catherine's Church, Cricieth', Caern. Hist. Soc. Trans., 58 (1997), 7-34 (10); Cartwright, Feminine Sanctity, t. 160. Cf. Lloyd, History of Wales, t. 690, lle nodir bod Llywelyn wedi rhoi croeso i'r Ffransisiaid, yn o fuan ar ôl i'w hurdd gyrraedd Prydain, ac mai o dan ei nawdd ef, yn ôl pob tebyg, yr ymsefydlodd Marchogion yr Ysbyty yn Nolgynwal neu Ysbyty Ifan, a leolir ger y Conwy ryw bedair milltir ar ddeg i’r de o Eglwys-bach.

158 Yn ôl Kenrick a Tucker, Eglwysbach, t. [4], roedd yr eglwys yn bodoli cyn amser Edward I.

159 Mae'n bosibl fod gweithiau Sulpicius ymhlith ffynonellau'r Historia Brittonum (gw. uchod, a n. 40), a cf. y copi cynnar ohonynt yn Llyfr Armagh (gw. Gwynn (gol.), Liber Ardmachanus, tt. cclix-cclxxviii, 377-438).

Cf. sylw Christine James fod Sant Lawrens 'yn gymaint rhan o ymwybyddiaeth grefyddol dyffryn Teifi yn y bymthegfed ganrif ag oedd y seintiau a ystyrir yn rhai lleol', yn 'Ysgrifydd Anhysbys: Proffil Personol', Ysgrifau Beirniadol, 23 (1997), tt. 44-72 (tt. 61-2). 